\title{
Facile Synthesis of Non-nucleoside Compounds Starting from $\alpha$-Chlorocarbenium Ions and Isocyanates as Potential HIV-1 Reverse Transcriptase Inhibitors
}

\author{
Atef A. Hamed, Ibrahim F. Zeid, and Alaa A. Manaa \\ Chemistry Department, Faculty of Science, Menoufia University, Shebin El Koam, Egypt \\ Reprint requests to Dr. Atef A. Hamed. Fax: +20-48-2235689. E-mail: atef2000_99@yahoo.com
}

Z. Naturforsch. 2009, 64b, 555-564; received February 12, 2009

Dedicated to Professor Volker Jaeger on the occasion of his $65^{\text {th }}$ birthday

\begin{abstract}
Chloro(phenyl)carbenium hexachloroantimonate salts react with isocyanates to afford either isoindolium (1) or benzoxazinium salts (2). Addition of one equivalent of alcohol to $\mathbf{2}$ led, after hydrolysis with aq. $\mathrm{NaOH}$, to the formation of benzoxazin-2-ones $\mathbf{3}$. Treatment with a large excess of alcohol transformed the salts $\mathbf{1}$ and $\mathbf{2}$ to the corresponding isoindol-1-ones $\mathbf{1 1}$ and the isocyanates $\mathbf{5}$, respectively. Reaction of $\mathbf{5}$ with primary amines furnished the urea derivatives $\mathbf{6}$ in good yield. The biological activity of $\mathbf{6 a}-\mathbf{0}$ against HIV-1 was determined.
\end{abstract}

Key words: $\alpha$-Chlorocarbenium Salts, Isocyanates, Isoindoles, Urea Derivatives, Benzoxa-zinones, Reverse Transcriptase Inhibitors

\section{Introduction}

The life cycle of HIV-1 has been extensively studied, and a number of stages identified for possible intervention to prevent viral replication. Clinically relevant agents which have been successfully developed are: a) nucleoside reverse transcriptase inhibitors (NRTIs), for example zidovudine (AZT), didanosine (DDI), zalcitabine (DDC), stavudine (D4T), lamivudine (3TC), abacavir (ABC), emtricitabine, and tenofovir disoproxil; b) non-nucleoside reverse transcriptase inhibitors (NNRTIs) such as ateviridine, capravirine, efavirenz, emivirine, lodenosine, nevirapine, etravirine, rilpivirine, loviride, delavirdine, and quinotaline; c) protease inhibitors (PIs) e. g., atazanavir, brecanavir, fosamprenavir, lopinavir, darunavir, nelfinavir, ritonavir, saquinavir, tipranavir, amprenavir, and indinavir; d) integrase inhibitors like raltegravir and elvitegravir; e) entry (fusion) inhibitors such as aplaviroc, enfuvirtide, maraviroc, vicriviroc, and ibalizumab; f) maturation inhibitors, for example bevirimat and vivecon. Several others are in preclinical or clinical development. From these compounds, thirty anti-HIV drugs have been approved and licensed for clinical use in the USA by the Food and Drug Administration (FDA). Four of them, namely, nevirapine [1,2], delavirdine [3, 4], efavirenz [5] and - the first to be ap- proved in 2008 - etravirine [6,7] belong to the group of NNRTIs. These compounds have gained a definitive place in the treatment of HIV-1 infections. Starting from HEPT [8] and TIBO [9] derivatives, more than 40 structurally different classes of compounds have been identified as NNRTIs. However, as with all current HIV therapies, drug incompatibilities, adverse effects, the emergence of resistant viral strains or of cross-resistance continue to limit the clinical usefulness of the NNRTIs. Therefore, additional NNRTIs are needed that might have improved pharmacokinetics, limited toxicities, and more favorable resistance mutation profiles.

In continuation of our previous work on searching antiviral drugs $[10,11]$, we report in this communication on a facile access to a new series of urea, isoindole and benzoxazine derivatives, analogs of troviridine [PETT (LY300046)] [12], thiazoloisoindolinone $(\mathrm{BM}+51.0836)$ [13], and efavirenz (DMP 266) [5] compounds.

\section{Results and Discussion}

The starting materials, 1-oxoisoindolium salts (1), could be prepared as described in the literature [14] from the reaction of alkyl- or electron-rich arylisocyanates with $\alpha$-chlorocarbenium salts, whereas the formation of benzoxazinium hexachloroantimon- 


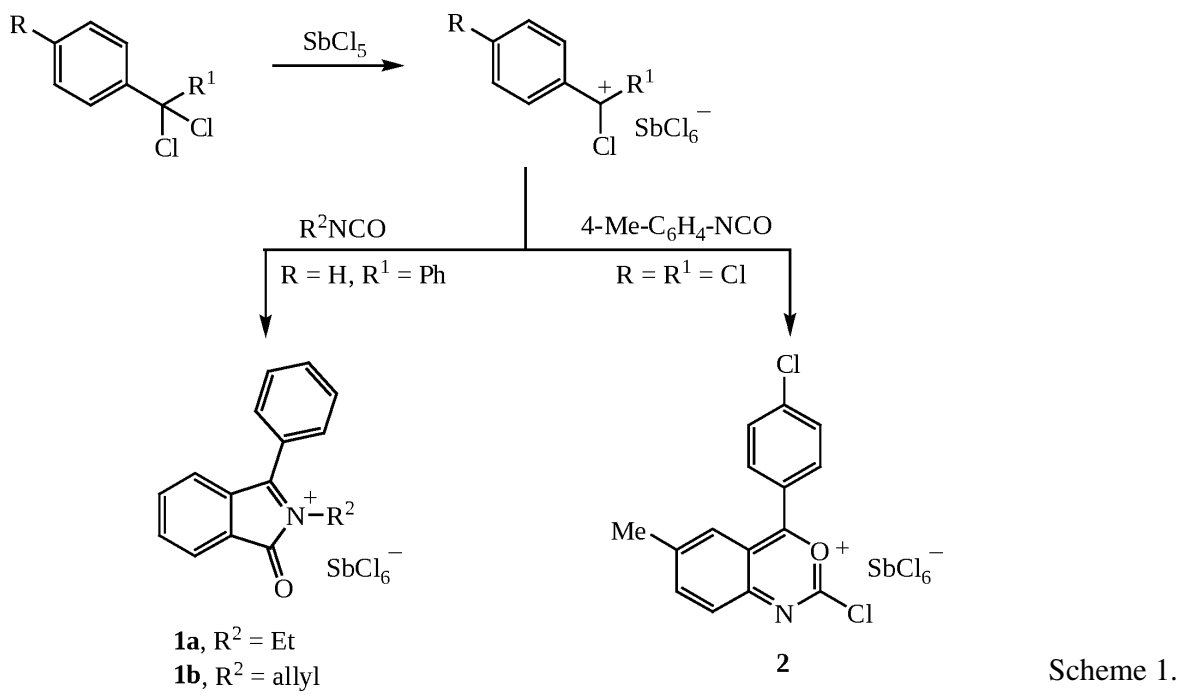

ate (2) resulted only from treatment of electrondeficient carbenium salts with electron-rich arylisocyanates (Scheme 1). For instance, addition of a solution of $\mathrm{SbCl}_{5}$ in $\mathrm{CH}_{2} \mathrm{Cl}_{2}$ to a cold $\left(-30{ }^{\circ} \mathrm{C}\right)$ mixture of $p$-tolylisocyanate and $p$-chlorobenzotrichloride resulted in the formation of $\mathbf{2}$. This salt is extremely sensitive toward nucleophiles. Dropwise addition of one molar equivalent of alcohol afforded, after hydrolysis with aq. $\mathrm{NaOH}, 3$,1-benzoxazin-2-ones $\mathbf{3}$, analogs of efavirenz. During purification of $\mathbf{3 b}$ by column chromatography on silica gel, using $15 \%$ EtOAc / $n$-hexane as eluent, the dibenzodiazocine 4 was isolated in $5 \%$ yield. The formation of $\mathbf{4}$ is probably due to condensation of two molar equivalents of 2 -amino- $4^{\prime}$-chloro4-methylbenzophenone which is formed as a result of partial hydrolysis of 2 [14].

Addition of a large excess of an alcohol in one portion to a cold $\left(-10{ }^{\circ} \mathrm{C}\right)$ suspension of 2 in absolute $\mathrm{CH}_{2} \mathrm{Cl}_{2}$ led to the formation of the isocyanates $\mathbf{5 a}, \mathbf{b}$ as yellow oils which could be used without further purification. Treatment of $\mathbf{5}$ with primary amines in warm diethyl ether gave the corresponding urea derivatives $\mathbf{6 a - 0}$ in good yield, analogs of urea-PETT compounds $[15,16]$. When 6 was exposed to a few drops of conc. $\mathrm{H}_{2} \mathrm{SO}_{4}$ or $\mathrm{SbCl}_{5}$ in $\mathrm{CH}_{2} \mathrm{Cl}_{2}$, quinazolinone 8 was obtained instead of the expected 4-alkoxy derivative 7 (Scheme 2).

The structure assignment of the prepared compounds is based, beside elemental analyses, on their spectral (IR, ${ }^{1} \mathrm{H},{ }^{13} \mathrm{C} \mathrm{NMR}$ ) data. The IR spectra of $1 \mathbf{b}$ (Nujol) showed absorption bands at 1757 and $1811 \mathrm{~cm}^{-1}$ for $\left(\mathrm{C}=\mathrm{N}^{+}\right)$and $(\mathrm{C}=\mathrm{O})$ groups, whereas compounds 3a, $\mathbf{b}$ exhibited bands in the ranges of $1720-1735$ and $3350-3413 \mathrm{~cm}^{-1}$ due to $\mathrm{C}=\mathrm{O}$ and NH absorptions, respectively. The urea derivatives $6 \mathbf{a}-$ o showed in their IR spectra $(\mathrm{KBr})$ absorption bands in the ranges $1664-1702$ and $3348-3380 \mathrm{~cm}^{-1}$ for $\mathrm{C}=\mathrm{O}$ and $(\mathrm{NH})$ groups. In the ${ }^{13} \mathrm{C} \mathrm{NMR}\left(\mathrm{CDCl}_{3}\right)$ spectra, the ketal carbons of $\mathbf{6 a}-\mathbf{o}$ resonated around $101.2 \mathrm{ppm}$.

Addition of a large excess of alcohol to a cold $\left(-10{ }^{\circ} \mathrm{C}\right)$ suspension of 3-phenyl-2-substituted-1-oxoisoindolium salts $\mathrm{I}$ in $\mathrm{CH}_{2} \mathrm{Cl}_{2}$ furnished 3-alkoxy derivatives 11a-f in moderate yield, whereas with traces of water or better aqueous base, 3-hydroxyisoindoles $(9 \mathbf{a}, \mathbf{b})$ were obtained. Reduction of the latter compounds by $\mathrm{H}_{2} / \mathrm{Pd} / \mathrm{C}$ in absolute methanol afforded isoindol-1-ones 10a, b (Scheme 3). The $N$-allyl group of $\mathbf{9 b}$ is converted to the $n$-propyl group during the reduction process. The constitutions of isoindoles 9, 10 and 11 were derived from spectroscopic data. IR spectra of $\mathbf{9 a}, \mathbf{b}$ showed absorption bands in the ranges $1686-1690$ and $3280-3290 \mathrm{~cm}^{-1}$ for $\mathrm{C}=\mathrm{O}$ and $\mathrm{OH}$ groups, whereas compounds $10 \mathbf{a}, \mathbf{b}$ and $11 \mathbf{a}-\mathbf{f}$ displayed bands in the range of $1674-1703 \mathrm{~cm}^{-1}$ for the carbonyl groups. In the ${ }^{1} \mathrm{H}$ NMR $\left(\mathrm{CDCl}_{3}\right)$ spectra of 9a, 10a and 11a-c diastereotopic $\mathrm{CH}_{2}$ protons were observed. The $\mathrm{OH}$ groups of compounds $\mathbf{9 a}, \mathbf{b}$ appeared in the range of $4.23-4.86 \mathrm{ppm}$, whereas the $3-\mathrm{H}$ protons of $\mathbf{1 0 a}, \mathbf{b}$ resonated as a singlet at $5.35-$ $5.46 \mathrm{ppm}$. The ${ }^{13} \mathrm{C}$ NMR spectra of $\mathbf{9}, \mathbf{1 0}$ and 11 displayed peaks for C-3 around 91.6, 64.2 and 94.6 ppm, respectively. The carbonyl carbons of the isoindole derivatives resonated between 167.0 and $168.3 \mathrm{ppm}$. 


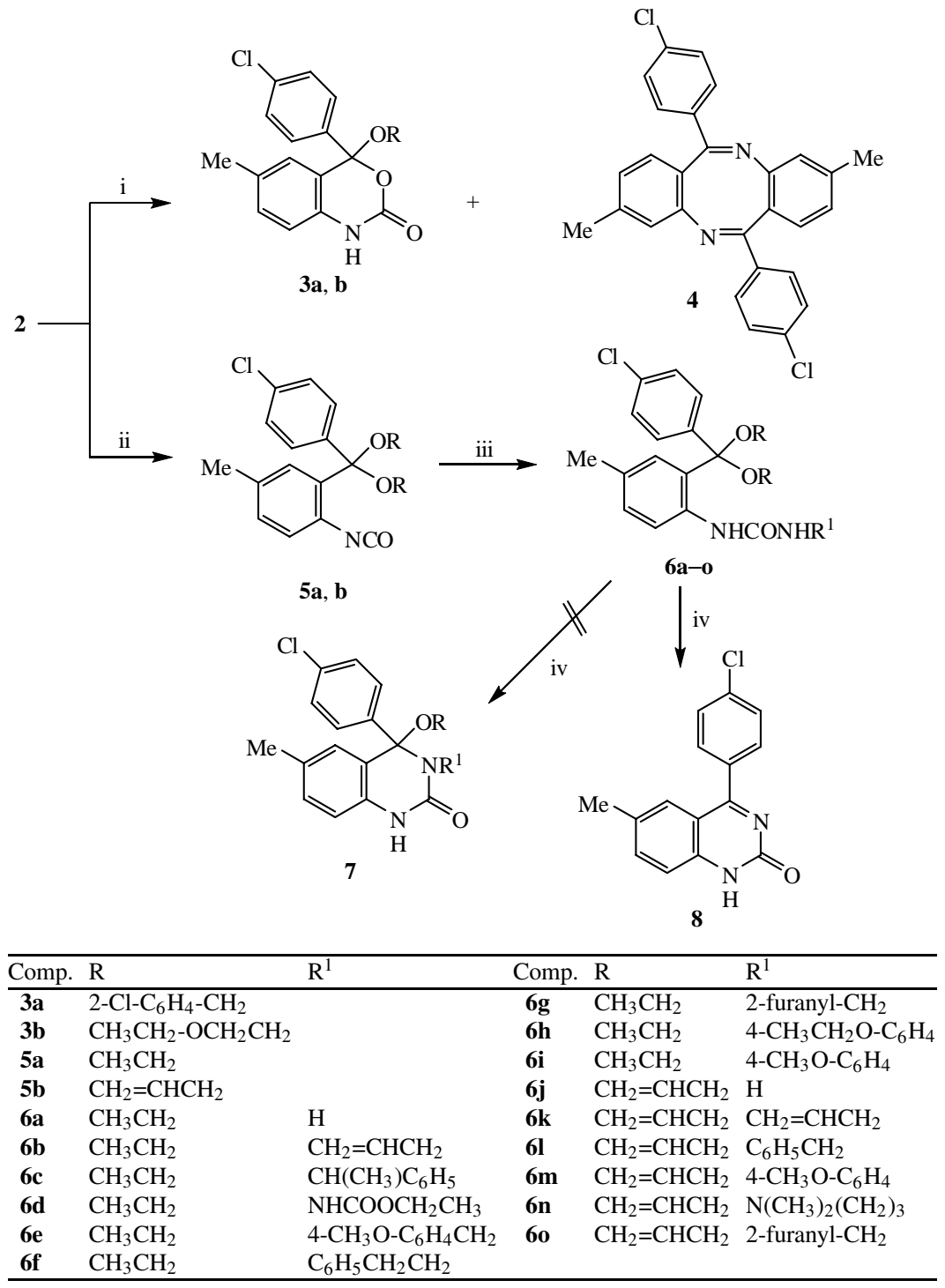

Scheme 2. Reagents and conditions: i) $\mathrm{ROH}, \quad \mathrm{CH}_{2} \mathrm{Cl}_{2}$, -30 to $23{ }^{\circ} \mathrm{C}$, aq. $\mathrm{NaOH}$, $20 \mathrm{~min}$; ii) large excess of $\mathrm{ROH},-10$ to $23{ }^{\circ} \mathrm{C}$, aq. $\mathrm{NaOH}$, 20 min; iii) $\mathrm{R}^{1} \mathrm{NH}_{2}, \mathrm{Et}_{2} \mathrm{O}, 35^{\circ} \mathrm{C}$, 5 min; iv) few drops of $\mathrm{SbCl}_{5}$ or $\mathrm{H}_{2} \mathrm{SO}_{4}, \mathrm{CH}_{2} \mathrm{Cl}_{2}, 35^{\circ} \mathrm{C}, 15 \mathrm{~min}$.

\section{Antiviral activity}

The urea compounds $\mathbf{6 a}-\mathbf{o}$ were examined for possible antiviral activity against the HIV-1 strain HTLVIIIB [17]. This strain of HIV-1 was propagated in $\mathrm{H} 9$ cells [18] at $37{ }^{\circ} \mathrm{C}, 5 \% \mathrm{CO}_{2}$, using RPMI 1640 with $10 \%$ heat-inactivated fetal calf serum (FCS) and antibiotics (growth medium). The culture supernatant was filtered $(0.45 \mathrm{~nm})$, aliquoted, and stored at $-80{ }^{\circ} \mathrm{C}$ until use. The MT-4 cells, which were used as target cells, were incubated with virus (0.005 MOI) for $2 \mathrm{~h}$, washed, and added in a proportion of $1: 10$ to uninfected cells which had been preincubated in growth medium containing the test compounds for $6 \mathrm{~d}$ in parallel with virus-infected control cultures without compound added. Expression of HIV in the culture medium was quantified by the HIV antigen detection assay ELISA [19].

Compounds $6 \mathbf{a}-\mathbf{o}$ did not exhibit any significant activity at non-toxic concentrations.

It was reported in the literature that phenylethylthiazolylthiourea (PETT, LY73497) shows high potency against HIV-1. Optimization of this lead compound gave $N$-[2-(2-pyridyl)ethyl- $N$-[2-(5-bromopyridyl)] thiourea (LY300046: $\mathrm{HCl}$ ) (troviridine) [12], which has been selected for clinical trials (Fig. 1). Ex- 


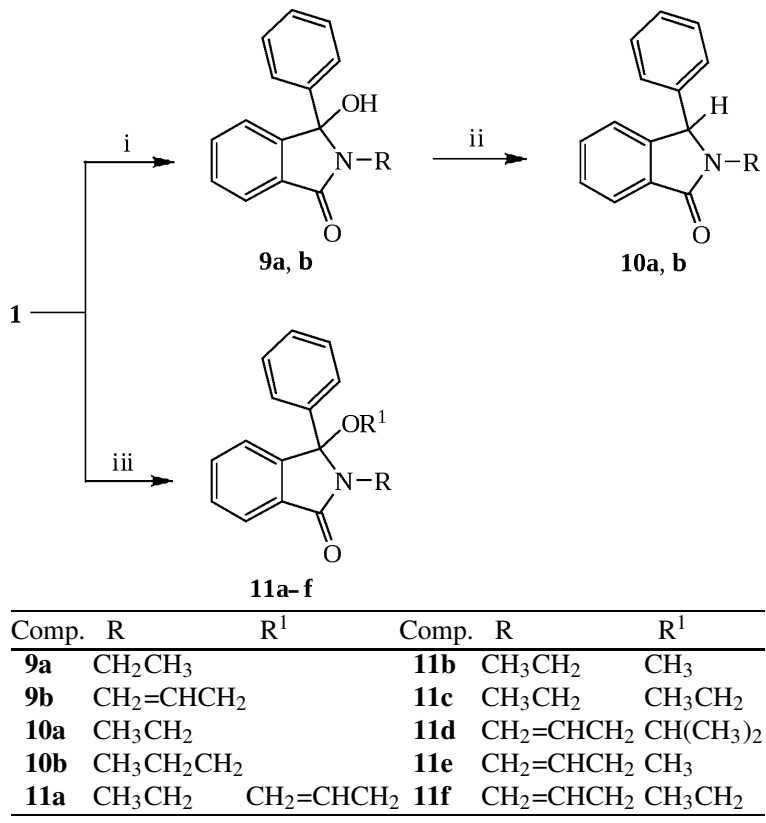

Scheme 3. Reagents and conditions: i) aq. $\mathrm{NaOH}$; ii) $\mathrm{H}_{2}$, $\mathrm{Pd} / \mathrm{C}, \mathrm{CH}_{3} \mathrm{OH}, 30 \mathrm{~min}$; iii) $\mathrm{R}^{1} \mathrm{OH},-10^{\circ} \mathrm{C}$, aq. $\mathrm{NaOH}$.

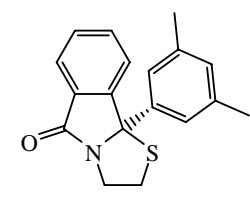

$\mathrm{BM}+51.0836$ Thiazoloisoindolinone

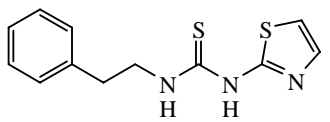

LY 73497 (PETT) phenyl ethyl thiazole thiourea

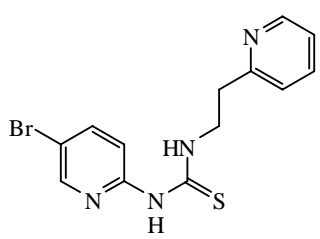

LY 300046 (PETT) Troviridine

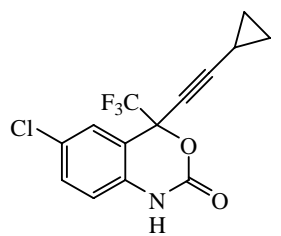

Efavirenz Sustiva (DMP 266)
Fig. 1. Selected non-nucleoside compounds in clinical use or development.

tensive structure-activity relationship (SAR) studies of the PETT compounds have been made [20,21]. For this purpose the structure of PETT is considered as a product of four parts (Fig. 2). In part 1 of the structure, mono-, di- and trisubstitutions gave very active compounds. Both electron donating and electron withdrawing small groups like chloro, fluoro, azido and methoxy substituents showed good activity. Compounds where

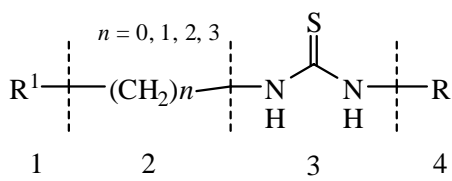

Fig. 2. The four parts of the PETT compounds.

the phenyl group was replaced with 2-pyridyl, as in troviridine, gave the most active compounds in a series with different heterocycles. In part 2, the ethyl linker was optimal for activity. In part 3, the $N, N$ unsubstituted thiourea was most active. Methyl substituents on nitrogen adjacent to the phenylethyl side chain completely eliminated the activity. In part 4 , the most optimal compound was achieved by replacing the thiazole with a 5-bromopyrid-2-yl. Later on, it was found that the urea-PETT compounds $[15,16]$ may have better toxicological and pharmacokinetic properties than the PETT compounds. Comparing these results with those for compounds $\mathbf{6 a - 0}$ leads us to believe that introducing the bulky group [dialkoxy( $p$ chlorophenyl)methyl] in position 2 of the tolyl substituent (part 4) is most likely the reason for elimination of the activity of compounds $6 \mathbf{a}-\mathbf{o}$.

\section{Experimental Section}

All solvents were dried by standard methods. All experiments were carried out with exclusion of moisture. Melting points were determined with a Kofler block apparatus and are uncorrected. IR spectra were recorded with a Perkin-Elmer Model 1720 FTIR spectrometer. ${ }^{1} \mathrm{H}$ and ${ }^{13} \mathrm{C}$ NMR spectra were determined with Varian Gemini 2000 and Brucker AC250 FT spectrometers. The chemical shifts in ppm are expressed on the $\delta$ scale using tetramethylsilane as internal standard. Coupling constants are given in Hz. Mass spectra were recorded on an AEIMS 30 spectrometer. TLC was performed on Merck silica gel 60-F254 precoated plastic plates. Microanalyses were performed in the unit of microanalysis at Cairo University (Egypt). The biological activity was determined in the Retrovirus Laboratory, State Serum Institute, Copenhagen (Denmark).

Synthesis of 2-allyl-1-oxo-3-phenyl-1H-isoindolium hexachloroantimonate $(\mathbf{1 b})$

A solution of $\mathrm{SbCl}_{5}(1.5 \mathrm{~g}, 5 \mathrm{mmol})$ in $\mathrm{CH}_{2} \mathrm{Cl}_{2}(5 \mathrm{~mL})$ was added dropwise with stirring at $-40{ }^{\circ} \mathrm{C}$ to a solution of dichlorodiphenylmethane $(1.2 \mathrm{~g}, 5 \mathrm{mmol})$ and allylisocyanate $(0.42 \mathrm{~g}, 6 \mathrm{mmol})$ in $\mathrm{CH}_{2} \mathrm{Cl}_{2}(15 \mathrm{~mL})$. The reaction mixture was warmed to $23^{\circ} \mathrm{C}$, and stirring was continued for $6 \mathrm{~h}$, upon which a yellowish orange precipitate was formed. Filtration, washing with $\mathrm{CH}_{2} \mathrm{Cl}_{2}(5 \mathrm{~mL})$ and drying under vac- 
cum afforded fine yellow crystals, m. p. $184-186{ }^{\circ} \mathrm{C}$; yield: $1.78 \mathrm{~g}(61 \%)$. - IR (Nujol): $v=1590(\mathrm{C}=\mathrm{C}), 1757\left(\mathrm{C}=\mathrm{N}^{+}\right)$, $1811 \mathrm{~cm}^{-1}(\mathrm{C}=\mathrm{O}) .-{ }^{1} \mathrm{H}$ NMR $\left(\mathrm{CD}_{3} \mathrm{CN}\right): \delta=4.74-4.76$ $\left(\mathrm{m}, 2 \mathrm{H}, \mathrm{NCH}_{2}\right), 5.43\left(\mathrm{dd}, 1 \mathrm{H}, J_{\text {cis }}=10.4 \mathrm{~Hz}, 3^{\prime}-\mathrm{H}_{\mathrm{a}}\right), 5.49$ $\left(\mathrm{dd}, 1 \mathrm{H}, J_{\text {trans }}=17.3 \mathrm{~Hz}, 3^{\prime}-\mathrm{H}_{\mathrm{b}}\right), 6.01-6.14\left(\mathrm{~m}, 1 \mathrm{H}, 2^{\prime}-\right.$ $\mathrm{H}), 7.66-8.25(\mathrm{~m}, 9 \mathrm{H}, \mathrm{Ar}-\mathrm{H}) .-{ }^{13} \mathrm{C} \mathrm{NMR}\left(\mathrm{CD}_{3} \mathrm{CN}\right): \delta=$ $47.2\left(\mathrm{NCH}_{2}\right), 116.9,134.8\left(\mathrm{CH}=\mathrm{CH}_{2}\right), 119.9,123.1,125.2$, 128.6, 129.5, 130.1, 132.0, 136.8, 138.1, 138.4, (Ar-C), 166.0, $184.2(\mathrm{C}=\mathrm{O}, \mathrm{C}=\mathrm{N})$ ppm. $-\mathrm{C}_{17} \mathrm{H}_{14} \mathrm{Cl}_{6} \mathrm{NOSb}(582.8)$ : calcd. C 35.03, H 2.43, N 2.40; found C 34.5, H 2.6, N 2.2.

General procedure for the preparation of 4,4-disubstituted 1,4-dihydro-benzo[d][1,3]oxazin-2-ones $\mathbf{3 a}, \boldsymbol{b}$

A solution of an alcohol $(7 \mathrm{mmol})$ in absolute $\mathrm{CH}_{2} \mathrm{Cl}_{2}$ $(10 \mathrm{~mL})$ was added to a cold $\left(-40{ }^{\circ} \mathrm{C}\right)$ suspension of 2 (5 mmol) in $\mathrm{CH}_{2} \mathrm{Cl}_{2}(50 \mathrm{~mL})$. The orange suspension disappeared immediately, and the reaction mixture became a clear yellow solution. Subsequently, a solution of $\mathrm{NaOH}(2 \mathrm{~N}$, $50 \mathrm{~mL}$ ) was added. After warming to r.t., the reaction mixture was stirred for $10 \mathrm{~min}$. The organic layer was separated, and the aqueous layer was repeatedly extracted with $\mathrm{CH}_{2} \mathrm{Cl}_{2}$. The combined organic extracts were dried over anhydrous $\mathrm{Na}_{2} \mathrm{SO}_{4}$. Filtration and evaporation of the solvent afforded a solid product, which could be recrystallized from appropriate solvents.

\section{4-(2-Chlorobenzyloxy)-4-(4-chlorophenyl)-6-methyl-1,4- dihydro-benzo[d][1,3]oxazin-2-one (3a)}

Prepared from 2-chlorobenzyl alcohol and $\mathbf{2}$ as described before. Recrystallization from $\mathrm{CHCl}_{3} / n$-pentane afforded a colorless powder; m. p. $192-193{ }^{\circ} \mathrm{C}$; yield: $1.83 \mathrm{~g}(63 \%)$. IR $(\mathrm{KBr}): v=1611(\mathrm{C}=\mathrm{C}), 1719(\mathrm{C}=\mathrm{O}), 3215 \mathrm{~cm}^{-1}(\mathrm{NH})$. ${ }^{1} \mathrm{H}$ NMR ([D 6 DMSO): $\delta=2.18\left(\mathrm{~s}, 1 \mathrm{H}, \mathrm{CH}_{3}\right), 4.60(\mathrm{~s}$, $\left.2 \mathrm{H}, \mathrm{OCH}_{2}\right), 6.80-7.53(\mathrm{~m}, 11 \mathrm{H}, \mathrm{Ar}-\mathrm{H}), 10.67(\mathrm{~s}, 1 \mathrm{H}$, $\mathrm{NH})$ ppm. $-\mathrm{C}_{22} \mathrm{H}_{17} \mathrm{Cl}_{2} \mathrm{NO}_{3}$ (414.3): calcd. C 63.78, $\mathrm{H} 4.14$, N 3.38; found C 63.4, H 3.8, N 3.0.

4-(4-Chlorophenyl)-4-(2-ethoxyethyloxy)-6-methyl-1,4dihydro-benzo[d][1,3]oxazin-2-one (3b)

From 2-ethoxyethanol and $\mathbf{2}$ as described for 3a. However, column chromatography on silica gel (EtOAc/ $n$-hexane, $1: 6)$ provided two fractions, which refer to compounds $\mathbf{3 b}$ $\left(R_{\mathrm{f}}=0.75\right)$ and $4\left(R_{\mathrm{f}}=0.55\right)$.

Compound $3 \boldsymbol{b}$ : pale-yellow powder, m.p. $175-177{ }^{\circ} \mathrm{C}$; yield: $1.57 \mathrm{~g}(62 \%)$. - IR (KBr): $v=1631,1590(\mathrm{C}=\mathrm{C})$, $1733(\mathrm{C}=\mathrm{O}), 3215 \mathrm{~cm}^{-1}(\mathrm{NH}) .-{ }^{1} \mathrm{H}$ NMR ([D 6$\left.] \mathrm{DMSO}\right)$ : $\delta=1.10\left(\mathrm{t}, 3 \mathrm{H}, J=7.2 \mathrm{~Hz}, \mathrm{CH}_{3}\right), 2.18\left(\mathrm{~s}, 3 \mathrm{H}, \mathrm{CH}_{3}\right), 3.45$ (q, $\left.2 \mathrm{H}, J=7.3 \mathrm{~Hz}, \mathrm{CH}_{2}\right), 3.54\left(\mathrm{~s}, 4 \mathrm{H}, 2 \mathrm{OCH}_{2}\right), 6.74(\mathrm{~s}, 1 \mathrm{H}$, Ar-H), $6.87-7.53$ (m, 6H, Ar-H), 10.77 (s, 1H, NH) ppm. $\mathrm{C}_{19} \mathrm{H}_{20} \mathrm{ClNO}_{4}$ (361.7): calcd. C 63.08, H 5.57, N 3.87; found C 62.7, H 5.2, N 3.5.
6,12-Bis(4-chlorophenyl)-3,9-dimethyldibenzo[b,f][1,5]diazocine (4)

Pale-yellow powder; m. p. $211^{\circ} \mathrm{C}$; yield: 0.16 g (5\%). ${ }^{1} \mathrm{H}$ NMR ([D $]$ DMSO): $\delta=2.20\left(\mathrm{~s}, 6 \mathrm{H}, 2 \mathrm{CH}_{3}\right), 6.82$ (s, 2H, Ar-H), 6.92-7.64 (m, 12H, Ar-H). $-{ }^{13} \mathrm{C}$ NMR $\left(\left[\mathrm{D}_{6}\right] \mathrm{DMSO}\right): \delta=20.1\left(2 \mathrm{CH}_{3}\right), 120.5,125.5,127.2$, 128.6, 130.3, 130.8, 133.0, 136.1, 148.5 (Ar-C), 167.5 $(2 \mathrm{C}=\mathrm{N})$ ppm. $-\mathrm{MS}(\mathrm{EI}, 70 \mathrm{eV}): \mathrm{m} / \mathrm{z}(\%)=454(100)[\mathrm{M}-$ $1]^{+} .-\mathrm{C}_{28} \mathrm{H}_{20} \mathrm{Cl}_{2} \mathrm{~N}_{2}$ (455.1): calcd. C 73.83, H 4.40, N 6.15; found $\mathrm{C} 73.5, \mathrm{H} 4.1$, N 5.7.

General procedure for the preparation of 4'-chloro-2isocyanato-5-methylbenzophenone ketals $5 \boldsymbol{a}, \boldsymbol{b}$

A solution of a large excess of alcohol $(3 \mathrm{~mL})$ in $\mathrm{CH}_{2} \mathrm{Cl}_{2}$ $(10 \mathrm{~mL})$ was added in one portion to a cold $\left(-10{ }^{\circ} \mathrm{C}\right)$ suspension of $2(5 \mathrm{mmol})$ in $\mathrm{CH}_{2} \mathrm{Cl}_{2}(40 \mathrm{~mL})$. After warming to r. t. in the course of $20 \mathrm{~min}$, a solution of $\mathrm{NaOH}(2 \mathrm{~g})$ in $\mathrm{H}_{2} \mathrm{O}$ $(50 \mathrm{~mL})$ was added. Usual work-up, drying over $\mathrm{Na}_{2} \mathrm{SO}_{4}$, filtration and evaporation of the solvent afforded a yellow oil which was used without further purification.

4'-Chloro-2-isocyanato-5-methylbenzophenone-diethylketal (5a)

From 2 and ethanol (3 mL) as described before [14].

\section{4'-Chloro-2-isocyanato-5-methylbenzophenone-diallylketal} (5b)

From 2 and allyl alcohol (3 mL); yield: $1.63 \mathrm{~g}(88 \%)$. IR (film): $v=1593(\mathrm{C}=\mathrm{C}), 2291 \mathrm{~cm}^{-1}(\mathrm{NCO}) .-{ }^{1} \mathrm{H}$ NMR $\left(\mathrm{CDCl}_{3}\right): \delta=2.36\left(\mathrm{~s}, 3 \mathrm{H}, \mathrm{CH}_{3}\right), 3.77\left(\mathrm{~m}, 4 \mathrm{H}, 2 \mathrm{OCH}_{2}\right), 5.14$ $\left(\mathrm{dd}, 2 \mathrm{H}, J_{\text {cis }}=10.3 \mathrm{~Hz}, 3^{\prime}-\mathrm{H}_{\mathrm{a}}\right), 5.36\left(\mathrm{dd}, 2 \mathrm{H}, J_{\text {trans }}=17.0 \mathrm{~Hz}\right.$, $\left.3^{\prime}-\mathrm{H}_{\mathrm{b}}\right), 5.90\left(\mathrm{~m}, 2 \mathrm{H}, 2^{\prime}-\mathrm{H}\right), 7.16-7.59(\mathrm{~m}, 7 \mathrm{H}, \mathrm{Ar}-\mathrm{H})$. $\mathrm{C}_{21} \mathrm{H}_{20} \mathrm{ClNO}_{3}$ (369.7): calcd. C 68.22, H 5.44, N 3.79; found C 68.5, H 5.3, N 3.5.

General procedure for the preparation of the derivatives of 1-\{2-[(4-chlorophenyl)-dialkyloxymethyl]-4-methylphenyl\}urea, $6 \boldsymbol{a}-\boldsymbol{o}$

A mixture of $5(5 \mathrm{mmol})$ and a primary amine $(8 \mathrm{mmol})$ in diethyl ether $(50 \mathrm{~mL})$ was boiled under reflux for $10 \mathrm{~min}$. The solid product that formed was filtered off and recrystallized from $\mathrm{CH}_{2} \mathrm{Cl}_{2} / \mathrm{Et}_{2} \mathrm{O}$ to give fine colorless crystals.

\section{1-\{2-[(4-Chlorophenyl)diethoxymethyl]-4-methylphenyl\}- urea $(6 a)$}

From ammonia and 5a as described before, m. p. 189 $191{ }^{\circ} \mathrm{C}$; yield: $1.60 \mathrm{~g}(88 \%)$. - IR $(\mathrm{KBr}): v=1592(\mathrm{C}=\mathrm{C})$, $1669(\mathrm{C}=\mathrm{O}), 3376 \mathrm{~cm}^{-1}(\mathrm{NH}) .-{ }^{1} \mathrm{H}$ NMR $\left(\mathrm{CDCl}_{3}\right): \delta=$ $1.21\left(\mathrm{t}, 6 \mathrm{H}, J=7.3 \mathrm{~Hz}, 2 \mathrm{CH}_{3}\right), 2.37\left(\mathrm{~s}, 3 \mathrm{H}, \mathrm{CH}_{3}\right), 3.23-$ $3.34\left(\mathrm{~m}, 4 \mathrm{H}, 2 \mathrm{OCH}_{2}\right), 4.32\left(\mathrm{~s}, 2 \mathrm{H}, \mathrm{NH}_{2}\right), 7.11-7.44(\mathrm{~m}, 7 \mathrm{H}$, 
$\mathrm{Ar}-\mathrm{H}), 7.71(\mathrm{~s}, 1 \mathrm{H}, \mathrm{NH}) .-{ }^{13} \mathrm{C} \mathrm{NMR}: \delta=15.3\left(2 \mathrm{CH}_{3}\right), 21.4$ $\left(\mathrm{CH}_{3}\right), 57.6\left(2 \mathrm{OCH}_{2}\right), 101.3(\mathrm{OCO}), 123.6,128.2,128.4$, 128.5, 128.9, 130.1, 132.3, 133.7, 134.0, 140.1 (Ar-C), 156.1 $(\mathrm{C}=\mathrm{O})$ ppm. $-\mathrm{C}_{19} \mathrm{H}_{23} \mathrm{ClN}_{2} \mathrm{O}_{3}$ (362.8): calcd. C 62.89, H 6.39, N 7.72; found C 62.6, H 6.8, N 7.9.

1-\{2-[(4-Chlorophenyl)diethoxymethyl]-4-methylphenyl $\}-3-$ allylurea $(\boldsymbol{6} \boldsymbol{b})$

From allylamine and 5a, m.p. $150-152{ }^{\circ} \mathrm{C}$; yield: $1.45 \mathrm{~g}(72 \%)$. - IR (KBr): $v=1594(\mathrm{C}=\mathrm{C}), 1661(\mathrm{C}=\mathrm{O})$, $3371 \mathrm{~cm}^{-1}(\mathrm{NH}) .-{ }^{1} \mathrm{H} \mathrm{NMR}\left(\mathrm{CDCl}_{3}\right): \delta=1.21(\mathrm{t}, 6 \mathrm{H}, J=$ $\left.7.3 \mathrm{~Hz}, 2 \mathrm{CH}_{3}\right), 2.37\left(\mathrm{~s}, 3 \mathrm{H}, \mathrm{CH}_{3}\right), 3.34\left(\mathrm{~m}, 4 \mathrm{H}, 2 \mathrm{OCH}_{2}\right)$, $3.60\left(\mathrm{~m}, 2 \mathrm{H}, \mathrm{NCH}_{2}\right), 4.11(\mathrm{t}, J=5,7 \mathrm{~Hz}, 1 \mathrm{H}, \mathrm{NH}), 4.95$ $\left(\mathrm{dd}, 1 \mathrm{H}, J_{\text {cis }}=10.4 \mathrm{~Hz}, 3^{\prime}-\mathrm{H}_{\mathrm{a}}\right), 5.02\left(\mathrm{dd}, 1 \mathrm{H}, J_{\text {trans }}=\right.$ $\left.17.2 \mathrm{~Hz}, 3^{\prime}-\mathrm{H}_{\mathrm{b}}\right), 5.59\left(\mathrm{~m}, 1 \mathrm{H}, 2^{\prime}-\mathrm{H}\right), 7.10-7.44(\mathrm{~m}, 7 \mathrm{H}$, $\mathrm{Ar}-\mathrm{H}), 7.45$ (s, 1H, NH). $-{ }^{13} \mathrm{C} \mathrm{NMR:} \delta=15.3\left(2 \mathrm{CH}_{3}\right), 21.4$ $\left(\mathrm{CH}_{3}\right), 42.9\left(\mathrm{NCH}_{2}\right), 57.5\left(2 \mathrm{OCH}_{2}\right), 101.1(\mathrm{OCO}), 115.8$, $133.7\left(\mathrm{CH}_{2}=\mathrm{CH}\right), 124.1,128.4,128.6,128.9,130.2,132.6$, 133.4, 134.1, 135.3, 140.2 (Ar-C), $155.3(\mathrm{C}=\mathrm{O}) \mathrm{ppm} .-$ $\mathrm{C}_{22} \mathrm{H}_{27} \mathrm{ClN}_{2} \mathrm{O}_{3}$ (402.9): calcd. C 65.58, H 6.75, N 6.95; found $\mathrm{C} 65.9, \mathrm{H} 7.1, \mathrm{~N} 7.2$.

1-\{2-[(4-Chlorophenyl)diethoxymethyl]-4-methylphenyl\}-3(1-phenylethyl)urea $(\boldsymbol{6 c})$

From 1-phenylethylamine and 5a, m.p. $199-201{ }^{\circ} \mathrm{C}$; yield: $1.66 \mathrm{~g}(71 \%)$. - IR (KBr): $v=1593(\mathrm{C}=\mathrm{C}), 1661$ $(\mathrm{C}=\mathrm{O}), 3370 \mathrm{~cm}^{-1}(\mathrm{NH}) .-{ }^{1} \mathrm{H}$ NMR $\left(\mathrm{CDCl}_{3}\right): \delta=1.15-$ $1.25\left(\mathrm{~m}, 6 \mathrm{H}, 2 \mathrm{CH}_{3}\right), 1.29\left(\mathrm{~d}, J=6.8 \mathrm{~Hz}, 3 \mathrm{H}, \mathrm{CH}_{3}\right), 2.36$ (s, $\left.3 \mathrm{H}, \mathrm{CH}_{3}\right), 3.19-3.34\left(\mathrm{~m}, 4 \mathrm{H}, 2 \mathrm{OCH}_{2}\right), 4.30(\mathrm{~m}, 1 \mathrm{H}, \mathrm{CH})$, $4.77(\mathrm{~s}, 1 \mathrm{H}, \mathrm{NH}), 7.07-7.43(\mathrm{~m}, 12 \mathrm{H}, \mathrm{Ar}-\mathrm{H}), 7.72(\mathrm{~s}, 1 \mathrm{H}$, $\mathrm{NH}) .-{ }^{13} \mathrm{C} \mathrm{NMR:} \delta=15.4\left(2 \mathrm{CH}_{3}\right), 21.4\left(\mathrm{CH}_{3}\right), 22.5\left(\mathrm{CH}_{3}\right)$, $49.6(\mathrm{NCH}), 57.6\left(2 \mathrm{OCH}_{2}\right), 101.1(\mathrm{OCO}), 123.8,126.3$, 127.4, 128.4, 128.6, 128.7, 128.9, 130.1, 132.2, 133.1, 133.8, 134.1, 140.3 (Ar-C), $154.5(\mathrm{C}=\mathrm{O})$ ppm. $-\mathrm{C}_{27} \mathrm{H}_{31} \mathrm{ClN}_{2} \mathrm{O}_{3}$ (466.9): calcd. C 69.45, H 6.66, N 6.00; found C 69.8, H 6.9, N 6.4.

Ethyl-2-\{2-[(4-chlorophenyl)diethoxymethyl]-4-methylphenylamino carbonylhydrazine carboxylate $(\boldsymbol{6 d})$

From ethyl carbamate and 5a, m.p. $203-205{ }^{\circ} \mathrm{C}$; yield: $1.51 \mathrm{~g}(67 \%)$. - IR (KBr): $v=1595(\mathrm{C}=\mathrm{C}), 1702(\mathrm{C}=\mathrm{O})$, $3352 \mathrm{~cm}^{-1}(\mathrm{NH}) .-{ }^{1} \mathrm{H} \mathrm{NMR}\left(\mathrm{CDCl}_{3}\right): \delta=1.18(\mathrm{t}, 6 \mathrm{H}, J=$ $\left.7.3 \mathrm{~Hz}, 2 \mathrm{CH}_{3}\right), 1.30$ (t, $\left.J=7.2 \mathrm{~Hz}, 3 \mathrm{H}, \mathrm{CH}_{3}\right), 2.37$ (s, $3 \mathrm{H}$, $\left.\mathrm{CH}_{3}\right), 3.23-3.34\left(\mathrm{~m}, 4 \mathrm{H}, 2 \mathrm{OCH}_{2}\right), 4.22(\mathrm{q}, J=6.8 \mathrm{~Hz}, 2 \mathrm{H}$, $\left.\mathrm{OCH}_{2}\right), 6.40,6.50(2 \mathrm{~s}, 2 \mathrm{H}, 2 \mathrm{NH}), 7.10-7.37(\mathrm{~m}, 7 \mathrm{H}, \mathrm{Ar}-\mathrm{H})$, $8.53(\mathrm{~s}, 1 \mathrm{H}, \mathrm{NH}) .-{ }^{13} \mathrm{C} \mathrm{NMR}: \delta=14.8\left(\mathrm{CH}_{3}\right), 15.2\left(2 \mathrm{CH}_{3}\right)$, $21.4\left(\mathrm{CH}_{3}\right), 57.6\left(2 \mathrm{OCH}_{2}\right), 62.9\left(\mathrm{OCH}_{2}\right), 101.3(\mathrm{OCO})$, 128.2, 128.3, 128.7, 128.8, 129.7, 130.0, 132.5, 133.1, 134.2, 139.7 (Ar-C), 155.6, $156.9(2 \mathrm{C}=\mathrm{O})$ ppm. $-\mathrm{C}_{22} \mathrm{H}_{28} \mathrm{ClN}_{3} \mathrm{O}_{5}$ (449.9): calcd. C 58.73, H 6.27, N 9.34; found C 59.1, H 6.6, N 9.7.
1-\{2-[(4-Chlorophenyl)diethoxymethyl]-4-methylphenyl\}-3(4-methoxybenzyl)urea (6e)

From 4-methoxybenzylamine and 5a, m.p. $190-192{ }^{\circ} \mathrm{C}$; yield: $1.71 \mathrm{~g}(71 \%)$. - IR (KBr): $v=1593(\mathrm{C}=\mathrm{C}), 1667$ $(\mathrm{C}=\mathrm{O}), 3368 \mathrm{~cm}^{-1}(\mathrm{NH}) .-{ }^{1} \mathrm{H}$ NMR $\left(\mathrm{CDCl}_{3}\right): \delta=1.19(\mathrm{t}$, $\left.6 \mathrm{H}, J=7.1 \mathrm{~Hz}, 2 \mathrm{CH}_{3}\right), 2.30\left(\mathrm{~s}, 3 \mathrm{H}, \mathrm{CH}_{3}\right), 3.18-3.32(\mathrm{~m}, 4 \mathrm{H}$, $\left.2 \mathrm{OCH}_{2}\right), 3.73\left(\mathrm{~s}, 3 \mathrm{H}, \mathrm{OCH}_{3}\right) 4.08\left(\mathrm{~d}, J=6.1 \mathrm{~Hz}, 2 \mathrm{H}, \mathrm{NCH}_{2}\right)$, $6.86-7.63(\mathrm{~m}, 11 \mathrm{H}, \operatorname{Ar}-\mathrm{H}), 7.71(\mathrm{~s}, 1 \mathrm{H}, \mathrm{NH}), 8.31(\mathrm{~s}, 1 \mathrm{H}$, $\mathrm{NH}) .-{ }^{13} \mathrm{C}$ NMR: $\delta=15.2\left(2 \mathrm{CH}_{3}\right), 21.6\left(\mathrm{CH}_{3}\right), 42.9$ $\left(\mathrm{NCH}_{2}\right), 55.9\left(\mathrm{OCH}_{3}\right), 57.7\left(\mathrm{OCH}_{2}\right), 101.3(\mathrm{OCO}), 114.5$, 123.4, 128.0, 128.6, 129.0, 129.2, 129.5, 129.8, 130.3, 131.1, 133.2, 135.0, 140.8, 159.0 (Ar-C), $155.3(\mathrm{C}=\mathrm{O}) \mathrm{ppm}$. $\mathrm{C}_{27} \mathrm{H}_{31} \mathrm{ClN}_{2} \mathrm{O}_{4}$ (483.0): calcd. C 67.14, H 6.47, N 5.80; found C 67.5, H 6.8, N 6.1.

1-\{2-[(4-Chlorophenyl)diethoxymethyl]-4-methylphenyl $\}-3-$ (2-phenylethyl)urea (6f)

From 2-phenylethylamine and 5a, m.p. $195-197{ }^{\circ} \mathrm{C}$; yield: $1.80 \mathrm{~g}(77 \%)$. - IR (KBr): $v=1593(\mathrm{C}=\mathrm{C}), 1655$ $(\mathrm{C}=\mathrm{O}), 3374 \mathrm{~cm}^{-1}(\mathrm{NH}) .-{ }^{1} \mathrm{H}$ NMR $\left(\mathrm{CDCl}_{3}\right): \delta=1.80$ (t, $\left.6 \mathrm{H}, J=7.3 \mathrm{~Hz}, 2 \mathrm{CH}_{3}\right), 2.37\left(\mathrm{~s}, 3 \mathrm{H}, \mathrm{CH}_{3}\right), 2.61(\mathrm{~m}, 2 \mathrm{H}$, $\mathrm{CH}_{2}$ ), 3.19-3.31 (m, 6H, 3CH$), 4.08$ (bs, $\left.1 \mathrm{H}, \mathrm{NH}\right), 7.01-$ $7.38(\mathrm{~m}, 12 \mathrm{H}, \mathrm{Ar}-\mathrm{H}), 7.70(\mathrm{~s}, 1 \mathrm{H}, \mathrm{NH}) .-{ }^{13} \mathrm{C} \mathrm{NMR}: \delta=$ $15.4\left(2 \mathrm{CH}_{3}\right), 21.4\left(\mathrm{CH}_{3}\right), 36.4\left(\mathrm{CH}_{2}\right), 41.7\left(\mathrm{NCH}_{2}\right), 57.6$ $\left(2 \mathrm{OCH}_{2}\right), 101.2(\mathrm{OCO}), 123.8,126.7,128.3,128.4,128.6$, 128.8, 128.9, 129.1, 130.1, 132.3, 133.2, 133.8, 134.0, 140.1 (Ar-C), $155.3(\mathrm{C}=\mathrm{O})$ ppm. $-\mathrm{C}_{27} \mathrm{H}_{31} \mathrm{ClN}_{2} \mathrm{O}_{3}$ (466.9): calcd. C 69.45, H 6.66, N 6.00; found C 69.7, H 6.8, N 6.2.

\section{1-\{2-[(4-Chlorophenyl)diethoxymethyl]-4-methylphenyl\}-3-} furan-2-yl-methylurea $(\mathbf{6 g})$

From 2-furanylmethylamine and 5a, m.p. $158-160{ }^{\circ} \mathrm{C}$; yield: $1.62 \mathrm{~g}(73 \%)$. - IR (KBr): $v=1594(\mathrm{C}=\mathrm{C}), 1661$ $(\mathrm{C}=\mathrm{O}), 3367 \mathrm{~cm}^{-1}(\mathrm{NH}) .-{ }^{1} \mathrm{H}$ NMR $\left(\mathrm{CDCl}_{3}\right): \delta=1.91$ (t, $\left.6 \mathrm{H}, J=7.3 \mathrm{~Hz}, 2 \mathrm{CH}_{3}\right), 2.36\left(\mathrm{~s}, 3 \mathrm{H}, \mathrm{CH}_{3}\right), 3.19-3.29$ (m, $\left.4 \mathrm{H}, 2 \mathrm{OCH}_{2}\right), 4.18\left(\mathrm{~d}, J=5.9 \mathrm{~Hz}, 2 \mathrm{H}, \mathrm{NCH}_{2}\right), 4.40$ (t, $1 \mathrm{H}$, $J=5.5 \mathrm{~Hz}, \mathrm{NH}), 6.04(\mathrm{~d}, J=2.5 \mathrm{~Hz}, 1 \mathrm{H}$, furanyl), $6.30(\mathrm{~m}$, $1 \mathrm{H}$, furanyl), $7.08-7.47(\mathrm{~m}, 7 \mathrm{H}, \mathrm{Ar}-\mathrm{H}), 7.72(\mathrm{~d}, J=1.8 \mathrm{~Hz}$, 1H, furanyl). $-{ }^{13} \mathrm{C}$ NMR: $\delta=15.4\left(2 \mathrm{CH}_{3}\right), 21.4\left(\mathrm{CH}_{3}\right), 37.5$ $\left(\mathrm{NCH}_{2}\right), 57.6\left(2 \mathrm{OCH}_{2}\right), 101.2(\mathrm{OCO}), 107.2,110.7,142.2$, 152.3 (furanyl-C), 123.8, 128.4, 128.5, 128.9, 130.2, 132.3, 133.3, 133.7, 134.0, 140.1 (Ar-C), $155.0(\mathrm{C}=\mathrm{O}) \mathrm{ppm}$. $\mathrm{C}_{24} \mathrm{H}_{27} \mathrm{ClN}_{2} \mathrm{O}_{4}$ (442.9): calcd. C 65.08, H 6.14, N 6.32; found $\mathrm{C} 64.7$, H 5.9, N 5.9

1-\{2-[(4-Chlorophenyl)diethoxymethyl]-4-methylphenyl\}-3(4-ethoxyphenyl)urea (6h)

From 4-ethoxyaniline and 5a, m.p. $199-201{ }^{\circ} \mathrm{C}$; yield: $1.93 \mathrm{~g}(80 \%)$. - IR (KBr): $v=1594(\mathrm{C}=\mathrm{C}), 1667(\mathrm{C}=\mathrm{O})$, $3371 \mathrm{~cm}^{-1}(\mathrm{NH}) .-{ }^{1} \mathrm{H} \mathrm{NMR}\left(\mathrm{CDCl}_{3}\right): \delta=1.11(\mathrm{t}, 6 \mathrm{H}, J=$ $\left.7.2 \mathrm{~Hz}, 2 \mathrm{CH}_{3}\right), 1.43\left(\mathrm{t}, 3 \mathrm{H}, J=7.1 \mathrm{~Hz}, \mathrm{CH}_{3}\right), 2.37$ (s, 3H, 
$\left.\mathrm{CH}_{3}\right), 3.13-3.25\left(\mathrm{~m}, 4 \mathrm{H}, 2 \mathrm{OCH}_{2}\right), 4.06(\mathrm{q}, 2 \mathrm{H}, J=7.1 \mathrm{~Hz}$, $\left.\mathrm{OCH}_{2}\right), 6.10(\mathrm{~s}, 1 \mathrm{H}, \mathrm{NH}), 6.86-7.71(\mathrm{~m}, 11 \mathrm{H}, \mathrm{Ar}-\mathrm{H})$. ${ }^{13} \mathrm{C} \mathrm{NMR:} \delta=15.3\left(2 \mathrm{CH}_{3}\right), 21.4\left(\mathrm{CH}_{3}\right), 57.4\left(2 \mathrm{OCH}_{2}\right), 64.1$ $\left(\mathrm{OCH}_{2}\right), 101.0(\mathrm{OCO}), 115.4,123.8,126.3,128.2,128.7$, 129.0, 130.0, 130.1, 131.6, 133.0, 133.5, 133.9, 139.6, 154.2 (Ar-C), $157.2(\mathrm{C}=\mathrm{O})$ ppm. $-\mathrm{C}_{27} \mathrm{H}_{31} \mathrm{ClN}_{2} \mathrm{O}_{4}$ (483.0): calcd. C 67.14, H 6.47, N 5.80; found C 67.4, H 6.7, N 6.2.

1-\{2-[(4-Chlorophenyl)diethoxymethyl]-4-methylphenyl\}-3(4-methoxyphenyl)urea (6i)

From 4-methoxyaniline and 5a, m. p. $150-152{ }^{\circ} \mathrm{C}$; yield: $1.88 \mathrm{~g}(80 \%)$. - IR (KBr): $v=1595(\mathrm{C}=\mathrm{C}), 1667(\mathrm{C}=\mathrm{O})$, $3370 \mathrm{~cm}^{-1}(\mathrm{NH}) .-{ }^{1} \mathrm{H}$ NMR $\left(\mathrm{CDCl}_{3}\right): \delta=1.17(\mathrm{t}, 6 \mathrm{H}$, $\left.J=7.2 \mathrm{~Hz}, 2 \mathrm{CH}_{3}\right), 2.37\left(\mathrm{~s}, 3 \mathrm{H}, \mathrm{CH}_{3}\right), 3.13-3.25(\mathrm{~m}, 4 \mathrm{H}$, $\left.2 \mathrm{OCH}_{2}\right), 3.83\left(\mathrm{~s}, 3 \mathrm{H}, \mathrm{OCH}_{3}\right), 5.96(\mathrm{~s}, 1 \mathrm{H}, \mathrm{NH}), 6.86-$ $7.25(\mathrm{~m}, 11 \mathrm{H}, \mathrm{Ar}-\mathrm{H}), 7.41(\mathrm{~s}, 1 \mathrm{H}, \mathrm{NH}),-{ }^{13} \mathrm{C} \mathrm{NMR}$ : $\delta=15.3\left(2 \mathrm{CH}_{3}\right), 21.4\left(\mathrm{CH}_{3}\right), 55.9\left(\mathrm{OCH}_{3}\right), 57.4\left(\mathrm{OCH}_{2}\right)$, 101.0 (OCO), 114.8, 123.8, 126.2, 128.2, 128.7, 130.0, 130.3, 131.6, 133.0, 133.5, 133.9, 139.7, 154.1 (Ar-C), 157.8 $(\mathrm{C}=\mathrm{O})$ ppm. $-\mathrm{C}_{26} \mathrm{H}_{29} \mathrm{ClN}_{2} \mathrm{O}_{4}$ (469.0): calcd. C 66.59, H 6.23, N 5.97; found C 66.9, H 6.5, N 6.3.

\section{1-\{2-[(4-Chlorophenyl)diallyloxymethyl]-4-methylphenyl\}- urea $(6 \mathbf{j})$}

From ammonia and $\mathbf{5 b}$, m.p. $155-156{ }^{\circ} \mathrm{C}$; yield: $1.61 \mathrm{~g}$ (83\%). - IR (KBr): $v=1591 \quad(\mathrm{C}=\mathrm{C}), 1669(\mathrm{C}=\mathrm{O})$, $3380 \mathrm{~cm}^{-1}(\mathrm{NH}) .-{ }^{1} \mathrm{H}$ NMR $\left(\mathrm{CDCl}_{3}\right): \delta=2.37(\mathrm{~s}, 3 \mathrm{H}$, $\left.\mathrm{CH}_{3}\right), 3.79\left(\mathrm{~m}, 4 \mathrm{H}, 2 \mathrm{OCH}_{2}\right), 4.50\left(\mathrm{~s}, 2 \mathrm{H}, \mathrm{NH}_{2}\right), 5.17$ $\left(\mathrm{dd}, 2 \mathrm{H}, J_{\text {cis }}=10.5 \mathrm{~Hz}, 3^{\prime}-\mathrm{H}_{\mathrm{a}}\right), 5.32\left(\mathrm{dd}, 2 \mathrm{H}, J_{\text {trans }}=\right.$ $\left.17.1 \mathrm{~Hz}, 3^{\prime}-\mathrm{H}_{\mathrm{b}}\right), 5.92\left(\mathrm{~m}, 2 \mathrm{H}, 2^{\prime}-\mathrm{H}\right), 7.12-7.51(\mathrm{~m}, 7 \mathrm{H}$, $\mathrm{Ar}-\mathrm{H}), 7.75(\mathrm{~s}, 1 \mathrm{H}, \mathrm{NH}) .-{ }^{13} \mathrm{C} \mathrm{NMR}: \delta=21.5\left(\mathrm{CH}_{3}\right)$, $63.3\left(2 \mathrm{OCH}_{2}\right), 101.7(\mathrm{OCO}), 117.0,133.7,\left(2 \mathrm{CH}_{2}=\mathrm{CH}\right)$, 117.1, 124.0, 128.5, 128.6, 128.9, 130.5, 131.1, 134.3, 134.4, 139.5(Ar-C), $156.0(\mathrm{C}=\mathrm{O})$ ppm. $-\mathrm{C}_{21} \mathrm{H}_{23} \mathrm{ClN}_{2} \mathrm{O}_{3}$ (386.9): calcd. C 65.20, H 5.99, N 7.24; found C 64.9, H 5.6, N 6.9.

\section{1-\{2-[(4-Chlorophenyl)diallyloxymethyl]-4-methylphenyl\}- 3-allylurea $(6 \boldsymbol{k})$}

From allylamine and 5b, m.p. $197-199{ }^{\circ} \mathrm{C}$; yield: $1.64 \mathrm{~g}(77 \%)$. - IR (KBr): $v=1563(\mathrm{C}=\mathrm{C}), 1642(\mathrm{C}=\mathrm{O})$, $3360 \mathrm{~cm}^{-1}(\mathrm{NH}) .-{ }^{1} \mathrm{H} \mathrm{NMR}\left(\mathrm{CDCl}_{3}\right): \delta=2.38(\mathrm{~s}, 3 \mathrm{H}$, $\left.\mathrm{CH}_{3}\right), 3.58-3.62\left(\mathrm{~m}, 2 \mathrm{H}, \mathrm{NCH}_{2}\right), 3.82\left(\mathrm{~m}, 4 \mathrm{H}, 2 \mathrm{OCH}_{2}\right)$, $4.13(\mathrm{bs}, 1 \mathrm{H}, \mathrm{NH}), 4.95\left(\mathrm{dd}, 2 \mathrm{H}, J_{\text {cis }}=9.5 \mathrm{~Hz}, 3^{\prime}-\mathrm{H}_{\mathrm{a}}\right)$, $5.04\left(\mathrm{dd}, 2 \mathrm{H}, J_{\text {trans }}=16.7 \mathrm{~Hz}, 3^{\prime}-\mathrm{H}_{\mathrm{b}}\right), 5.17\left(\mathrm{dd}, 1 \mathrm{H}, J_{\text {cis }}=\right.$ $\left.10.4 \mathrm{~Hz}, 3^{\prime \prime}-\mathrm{H}_{\mathrm{a}}\right), 5.50\left(\mathrm{dd}, 1 \mathrm{H}, J_{\text {trans }}=16.7 \mathrm{~Hz}, 3^{\prime \prime}-\mathrm{H}_{\mathrm{b}}\right)$, $5.62\left(\mathrm{~m}, 2 \mathrm{H}, 2^{\prime}-\mathrm{H}\right), 5.89\left(\mathrm{~m}, 1 \mathrm{H}, 2^{\prime \prime}-\mathrm{H}\right), 7.07-7.49(\mathrm{~m}, 7 \mathrm{H}$, $\mathrm{Ar}-\mathrm{H}), 7.77$ (s, 1H, NH). $-{ }^{13} \mathrm{C}$ NMR: $\delta=21.5\left(\mathrm{CH}_{3}\right), 42.9$ $\left(\mathrm{NCH}_{2}\right), 63.3\left(2 \mathrm{OCH}_{2}\right), 101.5(\mathrm{OCO}), 115.8,117.1$. 133.7, $134.3\left(3 \mathrm{CH}_{2}=\mathrm{CH}\right), 124.3,128.5,128.6,128.8,130.4,131.9$, 133.5, 134.4, 135.3, 139.5 (Ar-C), $155.1(\mathrm{C}=\mathrm{O})$ ppm. $\mathrm{C}_{24} \mathrm{H}_{27} \mathrm{ClN}_{2} \mathrm{O}_{3}$ (426.9): calcd. C 67.52, H 6.37, N 6.56; found C 67.2, H 6.0, N 6.3.
1-\{2-[(4-Chlorophenyl)diallyloxymethyl]-4-methylphenyl\}3-benzylurea $(6 \mathbf{l})$

From benzylamine and $\mathbf{5 b}$, m.p. $193-194{ }^{\circ} \mathrm{C}$; yield: $1.88 \mathrm{~g}(79 \%)$. - IR (KBr): $v=1592(\mathrm{C}=\mathrm{C}), 1657(\mathrm{C}=\mathrm{O})$, $3369 \mathrm{~cm}^{-1}(\mathrm{NH}) .-{ }^{1} \mathrm{H} \mathrm{NMR}\left(\mathrm{CDCl}_{3}\right): \delta=2.36(\mathrm{~s}, 3 \mathrm{H}$, $\left.\mathrm{CH}_{3}\right), 3.78\left(\mathrm{~m}, 4 \mathrm{H}, 2 \mathrm{OCH}_{2}\right), 4.20(\mathrm{~d}, 2 \mathrm{H}, J=5.7 \mathrm{~Hz}$, $\left.\mathrm{NCH}_{2}\right), 4.38(\mathrm{~s}, 1 \mathrm{H}, \mathrm{NH}), 5.15\left(\mathrm{dd}, 2 \mathrm{H}, J_{\text {cis }}=10.9 \mathrm{~Hz}\right.$, $\left.3^{\prime}-\mathrm{H}_{\mathrm{a}}\right), 5.30\left(\mathrm{dd}, 2 \mathrm{H}, J_{\text {trans }}=17.6 \mathrm{~Hz}, 3^{\prime}-\mathrm{H}_{\mathrm{b}}\right), 5.82(\mathrm{~m}, 2 \mathrm{H}$, $\left.2^{\prime}-\mathrm{H}\right), 7.07-7.54(\mathrm{~m}, 12 \mathrm{H}, \mathrm{Ar}-\mathrm{H}) .-{ }^{13} \mathrm{C}$ NMR: $\delta=21.2$ $\left(\mathrm{CH}_{3}\right), 44.3\left(\mathrm{NCH}_{2}\right), 63.1\left(2 \mathrm{OCH}_{2}\right), 101.3(\mathrm{OCO}), 116.9$, 134.1 $\left(2 \mathrm{CH}_{2}=\mathrm{CH}\right), 123.7,127.4,127.5,128.3,128.4,128.6$, 128.7, 130.3, 131.3, 133.1, 133.5, 134.2, 139.3 (Ar-C), 154.9 $(\mathrm{C}=\mathrm{O})$ ppm. $-\mathrm{C}_{28} \mathrm{H}_{29} \mathrm{ClN}_{2} \mathrm{O}_{3}$ (477.0): calcd. C 70.50, H 6.13, N 5.87; found C 70.7, H 6.5, N 6.1.

\section{1-\{2-[(4-Chlorophenyl)diallyloxymethyl]-4-methylphenyl\}- 3-(4-methoxyphenyl)urea (6m)}

From 4-methoxyaniline and 5b, m. p. $206-208{ }^{\circ} \mathrm{C}$; yield: $1.90 \mathrm{~g}(77 \%)$. - IR (KBr): $v=1594(\mathrm{C}=\mathrm{C}), 1665(\mathrm{C}=\mathrm{O})$, $3381 \mathrm{~cm}^{-1}(\mathrm{NH}) .-{ }^{1} \mathrm{H} \mathrm{NMR}\left(\mathrm{CDCl}_{3}\right): \delta=2.37(\mathrm{~s}, 3 \mathrm{H}$, $\left.\mathrm{CH}_{3}\right), 3.71-3.77\left(\mathrm{~m}, 4 \mathrm{H}, 2 \mathrm{OCH}_{2}\right), 3.83$ (s, 3H, $\left.\mathrm{OCH}_{3}\right), 5.14$ $\left(\mathrm{dd}, 2 \mathrm{H}, J_{\text {cis }}=9.2 \mathrm{~Hz}, 3^{\prime}-\mathrm{H}_{\mathrm{a}}\right), 5.78\left(\mathrm{dd}, 2 \mathrm{H}, J_{\text {trans }}=15.5 \mathrm{~Hz}\right.$, $\left.3^{\prime}-\mathrm{H}_{\mathrm{b}}\right), 5.84\left(\mathrm{~m}, 2 \mathrm{H}, 2^{\prime}-\mathrm{H}\right), 6.86-7.38(\mathrm{~m}, 11 \mathrm{H}, \mathrm{Ar}-\mathrm{H}), 7.74$ (s, $1 \mathrm{H}, \mathrm{NH}) .-{ }^{13} \mathrm{C}$ NMR: $\delta=21.5\left(\mathrm{CH}_{3}\right), 55.8\left(\mathrm{OCH}_{3}\right)$, $63.2\left(2 \mathrm{OCH}_{2}\right), 101.3(\mathrm{OCO}), 116.9,134.7\left(2 \mathrm{CH}_{2}=\mathrm{CH}\right)$, 114.9, 123.8, 126.1, 128.4, 128.6, 130.2, 130.3, 130.8, 133.1, 133.5, 134.3, 139.0, 157.7 (Ar-C), $154.0(\mathrm{C}=\mathrm{O})$ ppm. $\mathrm{C}_{28} \mathrm{H}_{29} \mathrm{ClN}_{2} \mathrm{O}_{4}$ (493.0): calcd. C 68.22, H 5.93, N 5.68; found C 68.6. H 6.3, N 5.9.

\section{1-\{2-[(4-Chlorophenyl)diallyloxymethyl]-4-methylphenyl\}- 3-(3-dimethylaminopropyl)urea (6n)}

From 3-(dimethylamino)propylamine and 5b, m. p. 148 $150{ }^{\circ} \mathrm{C}$; yield: $(1.77 \mathrm{~g}(75 \%) .-\mathrm{IR}(\mathrm{KBr}): v=1570(\mathrm{C}=\mathrm{C})$, $1640(\mathrm{C}=\mathrm{O}), 3348 \mathrm{~cm}^{-1}(\mathrm{NH}) .-{ }^{1} \mathrm{H} \mathrm{NMR}\left(\mathrm{CDCl}_{3}\right): \delta=$ $1.41-1.50\left(\mathrm{~m}, 2 \mathrm{H}, \mathrm{CH}_{2}\right), 2.07\left(\mathrm{~s}, 6 \mathrm{H}, \mathrm{N}\left(\mathrm{CH}_{3}\right)_{2}\right), 2.10-$ $2.24\left(\mathrm{~m}, 2 \mathrm{H}, \mathrm{NCH}_{2}\right), 2.37\left(\mathrm{~s}, 3 \mathrm{H}, \mathrm{CH}_{3}\right), 3.02-3.08(\mathrm{~m}$, $\left.2 \mathrm{H}, \mathrm{NCH}_{2}\right), 3.79-3.85\left(\mathrm{~m}, 4 \mathrm{H}, 2 \mathrm{OCH}_{2}\right), 5.16(\mathrm{dd}, 2 \mathrm{H}$, $\left.J_{\text {cis }}=9.6 \mathrm{~Hz}, 3^{\prime}-\mathrm{H}_{\mathrm{a}}\right), 5.35\left(\mathrm{dd}, 2 \mathrm{H}, J_{\text {trans }}=17.1 \mathrm{~Hz}, 3^{\prime}-\mathrm{H}_{\mathrm{b}}\right)$, $5.66(\mathrm{t}, 1 \mathrm{H}, J=4.2 \mathrm{~Hz}, \mathrm{NH}), 5.84-5.97\left(\mathrm{~m}, 2 \mathrm{H}, 2^{\prime}-\mathrm{H}\right)$, $7.10-7.45$ (m, 7H, Ar-H), 7.77 (s, 1H, NH). $-{ }^{13} \mathrm{C}$ NMR: $\delta=21.4\left(\mathrm{CH}_{3}\right), 26.3\left(\mathrm{CH}_{2}\right), 40.7\left(\mathrm{NCH}_{2}\right), 45.7\left(\mathrm{~N}\left(\mathrm{CH}_{3}\right)_{2}\right)$, $59.1\left(\mathrm{NCH}_{2}\right), 63.2\left(2 \mathrm{OCH}_{2}\right), 101.3(\mathrm{OCO}), 116.9,134.3$ $\left(2 \mathrm{CH}_{2}=\mathrm{CH}\right), 124.2,128.4,128.6,128.7,130.3,131.6,133.0$, 134.1, 134.2, $139.5(\mathrm{Ar}-\mathrm{C}), 155.6(\mathrm{C}=\mathrm{O}) \mathrm{ppm} .-\mathrm{C}_{26} \mathrm{H}_{34}$ $\mathrm{ClN}_{3} \mathrm{O}_{3}$ (472.0): calcd. C 66.16, H 7.26, $\mathrm{N} \mathrm{8.90;} \mathrm{found}$ C 65.9, H 6.9, N 8.6.

\section{1-\{2-[(4-Chlorophenyl)diallyloxymethyl]-4-methylphenyl $\}$ - 3-furan-2-yl-methylurea (6o)}

From 2-furanylmethylamine and 5b, m.p. $166-168{ }^{\circ} \mathrm{C}$; yield: $1.84 \mathrm{~g}(79 \%)$. - IR (KBr): $v=1594(\mathrm{C}=\mathrm{C}), 1665$ 
$(\mathrm{C}=\mathrm{O}), 3361 \mathrm{~cm}^{-1}(\mathrm{NH}) .-{ }^{1} \mathrm{H} \mathrm{NMR}\left(\mathrm{CDCl}_{3}\right): \delta=2.37$ $\left(\mathrm{s}, 3 \mathrm{H}, \mathrm{CH}_{3}\right), 3.80\left(\mathrm{~m}, 4 \mathrm{H}, 2 \mathrm{OCH}_{2}\right), 4.17\left(\mathrm{~m}, 2 \mathrm{H}, \mathrm{NCH}_{2}\right)$, $4.40(\mathrm{bs}, 1 \mathrm{H}, \mathrm{NH}), 5.18\left(\mathrm{dd}, 2 \mathrm{H}, J_{\text {cis }}=10.0 \mathrm{~Hz}, 3^{\prime}-\mathrm{H}_{\mathrm{a}}\right)$, $5.35\left(\mathrm{dd}, 2 \mathrm{H}, J_{\text {trans }}=17.0 \mathrm{~Hz}, 3^{\prime}-\mathrm{H}_{\mathrm{b}}\right), 5.91\left(\mathrm{~m}, 2 \mathrm{H}, 2^{\prime}-\mathrm{H}\right)$, $6.05(\mathrm{~d}, J=3.1 \mathrm{~Hz}, 1 \mathrm{H}$, furanyl), $6.30(\mathrm{~d}, J=2.0 \mathrm{~Hz}, 1 \mathrm{H}$, furanyl), 7.11-7.51 (m, 7H, Ar-H), $7.74(\mathrm{~s}, 1 \mathrm{H}, \mathrm{NH})$. ${ }^{13} \mathrm{C}$ NMR: $\delta=21.5\left(\mathrm{CH}_{3}\right), 37.6\left(\mathrm{NCH}_{2}\right), 63.3\left(2 \mathrm{OCH}_{2}\right)$, 101.6 (OCO), 107.2, 107.3, 142.2, 152.3 (furanyl-C), 117.1, $134.3\left(2 \mathrm{CH}_{2}=\mathrm{CH}\right), 124.0,128.6,130.5,131.6,133.5,133.7$, 134.4, 139.5 (Ar-C), $155.9(\mathrm{C}=\mathrm{O}) \mathrm{ppm} .-\mathrm{C}_{26} \mathrm{H}_{27} \mathrm{ClN}_{2} \mathrm{O}_{4}$ (467.0): calcd. C 66.88, H 5.83, N 6.00; found C 66.5, H 5.5, N 6.4 .

\section{4-(4-Chlorophenyl)-6-methyl-1H-quinazolin-2-one (8)}

A solution of conc. $\mathrm{H}_{2} \mathrm{SO}_{4}$ or $\mathrm{SbCl}_{5}$ (3 drops) in $5 \mathrm{~mL}$ of $\mathrm{CH}_{2} \mathrm{Cl}_{2}$ was added to a solution of $6(5 \mathrm{mmol})$ in $\mathrm{CH}_{2} \mathrm{Cl}_{2}$ $(15 \mathrm{~mL})$. Boiling the reaction mixture under reflux for $10 \mathrm{~min}$ with stirring afforded a yellow precipitate which was recrystallized from ethanol to give fine yellow crystals, m. p. $205-$ $207{ }^{\circ} \mathrm{C}$; yield: $0.96 \mathrm{~g}(71 \%) .-{ }^{1} \mathrm{H}$ NMR ([D $]$ DMSO): $\delta=$ 2.32 (s, $\left.3 \mathrm{H}, \mathrm{CH}_{3}\right), 7.37-7.75(\mathrm{~m}, 7 \mathrm{H}, \mathrm{Ar}-\mathrm{H}), 9.17$ (bs, $1 \mathrm{H}$, $\mathrm{NH}) .-{ }^{13} \mathrm{C}$ NMR: $\delta=21.4\left(\mathrm{CH}_{3}\right), 114.8,116.9,129.1$, $129.7,132.4,133.2,133.9,137.3,140.3,143.4$ (Ar-C), 152.7, $174.1(\mathrm{C}=\mathrm{O}, \mathrm{C}=\mathrm{N})$ ppm. $-\mathrm{C}_{15} \mathrm{H}_{11} \mathrm{ClN}_{2} \mathrm{O}(270.7)$ : calcd. C 66.55, H 4.07, N 10.34; found C 66.9, H 4.4, N 10.7.

\section{General procedure for the preparation of $9 \boldsymbol{a}, \boldsymbol{b}$}

An aqueous solution of $\mathrm{NaOH}(2 \mathrm{~N}, 50 \mathrm{~mL})$ was added to a suspension of $\mathbf{1}(5 \mathrm{mmol})$ in $\mathrm{CH}_{2} \mathrm{Cl}_{2}(20 \mathrm{~mL})$ at $0{ }^{\circ} \mathrm{C}$ with stirring for $20 \mathrm{~min}$. The organic layer was separated, and the aqueous layer was repeatedly extracted with $\mathrm{CH}_{2} \mathrm{Cl}_{2}$. The combined extract was dried over anhydrous $\mathrm{Na}_{2} \mathrm{SO}_{4}$. Evaporation of the solvent afforded a colorless powder, which can be recrystallized from $\mathrm{CH}_{2} \mathrm{Cl}_{2} / n$-pentane to give fine colorless crystals.

\section{2-Ethyl-3-hydroxy-3-phenyl-2,3-dihydro-isoindol-1-one (9a)}

From 1a as described before, m.p. $157{ }^{\circ} \mathrm{C}$; yield: $1.15 \mathrm{~g}(91 \%)$. - IR (KBr): $v=1620(\mathrm{C}=\mathrm{C}), 1687(\mathrm{C}=\mathrm{O})$, $3325 \mathrm{~cm}^{-1}(\mathrm{OH}) .-{ }^{1} \mathrm{H} \mathrm{NMR}\left(\mathrm{CDCl}_{3}\right): \delta=0.94(\mathrm{t}, 3 \mathrm{H}, J=$ $\left.7.3 \mathrm{~Hz}, \mathrm{CH}_{3}\right), 2.97-3.09,3.33-3.45\left(2 \mathrm{~m}, 2 \mathrm{H}, \mathrm{NCH}_{2}\right), 4.23$ (s, 1H, OH), $7.23-7.58(\mathrm{~m}, 9 \mathrm{H}, \mathrm{Ar}-\mathrm{H}) .-{ }^{13} \mathrm{C}$ NMR: $\delta=$ $14.4\left(\mathrm{CH}_{3}\right), 34.5\left(\mathrm{NCH}_{2}\right), 91.7(\mathrm{C}-3), 122.9,123.4,126.6$, 128.6, 128.7, 129.7, 130.9, 132.8, 139.1, 149.4 (Ar-C), 168.0 $(\mathrm{C}=\mathrm{O})$ ppm. $-\mathrm{C}_{16} \mathrm{H}_{15} \mathrm{NO}_{2}$ (253.3): calcd. C 75.87, H 5.97, N 5.53; found C 75.5, H 5.6, N 5.3.

\section{2-Allyl-3-hydroxy-3-phenyl-2,3-dihydro-isoindol-1-one (9b)}

From 1b as described before, m.p. $142{ }^{\circ} \mathrm{C}$ (lit. [22]: $\left.145{ }^{\circ} \mathrm{C}\right)$; yield: $1.11 \mathrm{~g}(84 \%)$. - IR (KBr): $v=1558(\mathrm{C}=\mathrm{C})$, $1687(\mathrm{C}=\mathrm{O}), 3334 \mathrm{~cm}^{-1}(\mathrm{OH}) .-{ }^{1} \mathrm{H}$ NMR $\left(\mathrm{CDCl}_{3}\right): \delta=$ $3.49-4.00\left(\mathrm{~m}, 2 \mathrm{H}, \mathrm{NCH}_{2}\right), 4.45(\mathrm{~s}, 1 \mathrm{H}, \mathrm{OH}), 4.86-4.99(\mathrm{~m}$, $\left.2 \mathrm{H}, 3^{\prime}-\mathrm{H}_{\mathrm{a}}, 3^{\prime}-\mathrm{H}_{\mathrm{b}}\right), 5.52-5.65\left(\mathrm{~m}, 1 \mathrm{H}, 2^{\prime}-\mathrm{H}\right), 7.22-7.79(\mathrm{~m}$, 9H, Ar-H). $-{ }^{13} \mathrm{C} \mathrm{NMR:} \delta=42.1\left(\mathrm{NCH}_{2}\right), 91.7(\mathrm{C}-3), 117.5$, $133.8\left(\mathrm{CH}_{2}=\mathrm{CH}\right), 123.1,123.5,126.7,128.6,128.7,129.6$, 130.6, 132.9, 139.1, 149.4 (Ar-C), $168.0(\mathrm{C}=\mathrm{O})$ ppm. $\mathrm{C}_{17} \mathrm{H}_{15} \mathrm{NO}_{2}$ (265.1): calcd. C 76.95, H 5.66, N 5.28; found C 76.6, H 5.3, N 4.9.

\section{General procedure for the preparation of $10 a, b$}

A stream of hydrogen gas was passed through a mixture of $9(2 \mathrm{mmol})$ and $\mathrm{Pd} / \mathrm{C}(0.16 \mathrm{~g})$ in absolute methanol $(20 \mathrm{~mL})$ with stirring at r. t. for $30 \mathrm{~min}$. Filtration and evaporation of the solvent afforded a colorless powder.

\section{2-Ethyl-3-phenyl-2,3-dihydro-isoindol-1-one (10a)}

From 9a as described before, m.p. $100{ }^{\circ} \mathrm{C}$ (lit. [23]: $\left.98{ }^{\circ} \mathrm{C}\right)$; yield: $0.44 \mathrm{~g}(93 \%)$. - IR $(\mathrm{KBr}): v=1612(\mathrm{C}=\mathrm{C})$, $1674 \mathrm{~cm}^{-1}(\mathrm{C}=\mathrm{O}) .-{ }^{1} \mathrm{H}$ NMR $\left(\mathrm{CDCl}_{3}\right): \delta=1.12(\mathrm{t}, 3 \mathrm{H}$, $\left.J=7.2 \mathrm{~Hz}, \mathrm{CH}_{3}\right), 2.93-3.00,3.93-4.00\left(2 \mathrm{~m}, 2 \mathrm{H}, \mathrm{NCH}_{2}\right)$, $5.46(\mathrm{~s}, 1 \mathrm{H}, \mathrm{CH}), 7.13-7.89(\mathrm{~m}, 9 \mathrm{H}, \mathrm{Ar}-\mathrm{H}) .-{ }^{13} \mathrm{C} \mathrm{NMR:} \delta=$ $13.5\left(\mathrm{CH}_{3}\right), 34.9\left(\mathrm{NCH}_{2}\right), 63.9(\mathrm{C}-3), 123.0,123.3,127.5$, 128.2, 128.6, 129.1, 131.6, 131.8, 137.1, 146.2 (Ar-C), 168.3 $(\mathrm{C}=\mathrm{O})$ ppm. $-\mathrm{C}_{16} \mathrm{H}_{15} \mathrm{NO}$ (237.1): calcd. C 80.99, H 6.33 N 5.90; found C 81.3, H 6.6, N 6.2.

\section{3-Phenyl-2-n-propyl-2,3-dihydro-isoindol-1-one (10b)}

From $9 \mathrm{~b}$ as described before, m. p. $95^{\circ} \mathrm{C}$ (lit. [23]: $93{ }^{\circ} \mathrm{C}$ ); yield: $0.43 \mathrm{~g}(85 \%)$. $-\mathrm{IR}(\mathrm{KBr}): v=1611(\mathrm{C}=\mathrm{C}), 1672 \mathrm{~cm}^{-1}$ $(\mathrm{C}=\mathrm{O}) .-{ }^{1} \mathrm{H} \mathrm{NMR}\left(\mathrm{CDCl}_{3}\right): \delta=0.70-0.78\left(\mathrm{~m}, 3 \mathrm{H}, \mathrm{CH}_{3}\right)$, $1.36-1.53\left(\mathrm{~m}, 2 \mathrm{H}, \mathrm{CH}_{2}\right), 2.70-2.88,3.71-3.87(2 \mathrm{~m}, 2 \mathrm{H}$, $\left.\mathrm{NCH}_{2}\right), 5.35(\mathrm{~s}, 1 \mathrm{H}, \mathrm{CH}), 7.01-7.79(\mathrm{~m}, 9 \mathrm{H}, \mathrm{Ar}-\mathrm{H})$. ${ }^{13} \mathrm{C}$ NMR: $\delta=11.2\left(\mathrm{CH}_{3}\right), 21.4\left(\mathrm{CH}_{2}\right), 41.7\left(\mathrm{NCH}_{2}\right)$, 64.3 (C-3), 122.9, 123.3, 127.4, 128.1, 128.5, 128.9, 131.5, 131.6, 137.0, 146.1 (Ar-C), $168.5(\mathrm{C}=\mathrm{O})$ ppm. $-\mathrm{C}_{17} \mathrm{H}_{17} \mathrm{NO}$ (251.0): calcd. C 81.28, H 6.78, N 5.58; found C 81.6, H 7.1, N 5.8 .

\section{General procedure for the preparation of $11 a-f$}

Excess alcohol $(2 \mathrm{~mL})$ was added to a cold $\left(-10{ }^{\circ} \mathrm{C}\right)$ suspension of $1(5 \mathrm{mmol})$ in $\mathrm{CH}_{2} \mathrm{Cl}_{2}(20 \mathrm{~mL})$ with stirring for $20 \mathrm{~min}$. Aqueous $\mathrm{NaOH}(2 \mathrm{~N}, 50 \mathrm{~mL})$ was added to the reaction mixture. Repeated extraction of the organic layer with $\mathrm{CH}_{2} \mathrm{Cl}_{2}$, filtration, drying over $\mathrm{Na}_{2} \mathrm{SO}_{4}$ and evaporation of the solvent afforded fine colorless crystals.

\section{3-Allyloxy-2-ethyl-3-phenyl-2,3-dihydro-isoindol-1-one} (11a)

From 1a and allyl alcohol as describe before, m. p. $132^{\circ} \mathrm{C}$; yield: $1.36 \mathrm{~g}(93 \%)$. - IR (KBr): $v=1582(\mathrm{C}=\mathrm{C}), 1697 \mathrm{~cm}^{-1}$ $(\mathrm{C}=\mathrm{O}) .-{ }^{1} \mathrm{H} \mathrm{NMR}\left(\mathrm{CDCl}_{3}\right): \delta=1.05(\mathrm{t}, 3 \mathrm{H}, J=7.2 \mathrm{~Hz}$, 
$\left.\mathrm{CH}_{3}\right), 3.07-3.22,3.66-3.76\left(2 \mathrm{~m}, 2 \mathrm{H}, \mathrm{NCH}_{2}\right), 3.42-3.54$ $\left(\mathrm{m}, 2 \mathrm{H}, \mathrm{OCH}_{2}\right), 5.12-5.36\left(\mathrm{~m}, 2 \mathrm{H}, 3^{\prime}-\mathrm{H}_{\mathrm{a}}, 3^{\prime}-\mathrm{H}_{\mathrm{b}}\right), 5.80(\mathrm{~m}$, $\left.1 \mathrm{H}, 2^{\prime}-\mathrm{H}\right), 7.12-7.91(\mathrm{~m}, 9 \mathrm{H}, \mathrm{Ar}-\mathrm{H}) .-{ }^{13} \mathrm{C}$ NMR: $\delta=13.2$ $\left(\mathrm{CH}_{3}\right), 34.0\left(\mathrm{NCH}_{2}\right), 63.2\left(\mathrm{OCH}_{2}\right), 94.9(\mathrm{C}-3), 116.0,133.4$ $\left(\mathrm{CH}_{2}=\mathrm{CH}\right), 122.7,122.9,126.0,127.9,128.1,129.3,129.7$, 132.0, 133.4, 138.6, 145.2 (Ar-C), $167.7(\mathrm{C}=\mathrm{O}) \mathrm{ppm}$. $\mathrm{C}_{19} \mathrm{H}_{19} \mathrm{NO}_{2}$ (293.4): calcd. C 77.79, H 6.53, N 4.77; found C 77.4, H 6.2, N 4.8.

\section{2-Ethyl-3-methoxy-3-phenyl-2,3-dihydro-isoindol-1-one} (11b)

From 1a and methyl alcohol, m.p. $112{ }^{\circ} \mathrm{C}$; yield: $1.26 \mathrm{~g}$ (94\%). - IR (KBr): $v=1612(\mathrm{C}=\mathrm{C}), 1701 \mathrm{~cm}^{-1}(\mathrm{C}=\mathrm{O})$. ${ }^{1} \mathrm{H} \mathrm{NMR}\left(\mathrm{CDCl}_{3}\right): \delta=0.92\left(\mathrm{t}, 3 \mathrm{H}, J=7.2 \mathrm{~Hz}, \mathrm{CH}_{3}\right), 3.87$ $\left(\mathrm{s}, 3 \mathrm{H}, \mathrm{OCH}_{3}\right), 3.01-3.08,3.29-3.36\left(2 \mathrm{~m}, 2 \mathrm{H}, \mathrm{NCH}_{2}\right)$, $7.00-7.77(\mathrm{~m}, 9 \mathrm{H}, \mathrm{Ar}-\mathrm{H}) .-{ }^{13} \mathrm{C}$ NMR: $\delta=12.3\left(\mathrm{CH}_{3}\right)$, $33.2\left(\mathrm{NCH}_{2}\right), 49.3\left(\mathrm{OCH}_{3}\right), 94.5(\mathrm{C}-3), 121.9,122.2,125.2$, 127.3, 128.5, 131.2, 131.3, 137.8, 144.2 (Ar-C), 167.5 $(\mathrm{C}=\mathrm{O})$ ppm. $-\mathrm{C}_{17} \mathrm{H}_{17} \mathrm{NO}_{2}$ (267.3): calcd. C 76.38, H 6.41, N 5.24; found $\mathrm{C} 76.1, \mathrm{H} 6.1, \mathrm{~N} 4.9$.

3-Ethoxy-2-ethyl-3-phenyl-2,3-dihydro-isoindol-1-one (11c)

From 1a and ethyl alcohol, m. p. $90-91^{\circ} \mathrm{C}$; yield: $1.29 \mathrm{~g}$ (92\%). - IR (KBr): $v=1611(\mathrm{C}=\mathrm{C}), 1702 \mathrm{~cm}^{-1}(\mathrm{C}=\mathrm{O})$. ${ }^{1} \mathrm{H}$ NMR $\left(\mathrm{CDCl}_{3}\right): \delta=1.05\left(\mathrm{t}, 3 \mathrm{H}, J=7.2 \mathrm{~Hz}, \mathrm{CH}_{3}\right), 1.20$ $\left(\mathrm{t}, 3 \mathrm{H}, J=7.0 \mathrm{~Hz}, \mathrm{CH}_{3}\right), 2.94-3.00,3.39-3.67(2 \mathrm{~m}, 2 \mathrm{H}$, $\left.\mathrm{NCH}_{2}\right), 3.13-3.25\left(2 \mathrm{~m}, 2 \mathrm{H}, \mathrm{OCH}_{2}\right), 7.11-7.88(\mathrm{~m}, 9 \mathrm{H}$, Ar-H). $-{ }^{13} \mathrm{C}$ NMR: $\delta=13.5,14.9\left(2 \mathrm{CH}_{3}\right), 34.2\left(\mathrm{NCH}_{2}\right)$, 58.1.3 $\left(\mathrm{OCH}_{2}\right), 94.9(\mathrm{C}-3), 123.1,126.3,127.3,128.3,128.4$, 129.4, 132.2, 139.1, 145.0 (Ar-C), $168.0(\mathrm{C}=\mathrm{O})$ ppm. $\mathrm{C}_{18} \mathrm{H}_{19} \mathrm{NO}_{2}$ (281.4): calcd. C 76.84, $\mathrm{H}$ 6.81, $\mathrm{N} 4.98$; found C 76.5, H 6.6, N 4.6.

2-Allyl-3-isopropyloxy-3-phenyl-2,3-dihydro-isoindol-1-one (11d)

From $1 \mathrm{~b}$ and isopropyl alcohol, m.p. $150{ }^{\circ} \mathrm{C}$; yield: $1.40 \mathrm{~g}$ (91\%). - IR (KBr): $v=1612(\mathrm{C}=\mathrm{C}), 1703 \mathrm{~cm}^{-1}(\mathrm{C}=\mathrm{O})$. ${ }^{1} \mathrm{H}$ NMR $\left(\mathrm{CDCl}_{3}\right): \delta=0.91\left(\mathrm{~d}, 3 \mathrm{H}, J=6.1 \mathrm{~Hz}, \mathrm{CH}_{3}\right)$, $1.41\left(\mathrm{~d}, 3 \mathrm{H}, J=6.1 \mathrm{~Hz}, \mathrm{CH}_{3}\right), 3.37-3.48(\mathrm{~m}, 1 \mathrm{H}, \mathrm{OCH})$,

[1] V.J. Merluzzi, K.D. Hargave, M. Labadia, K. Grozinger, M. Skoog, J.C. Wu, C. K. Shih, K. Eckner, S. Hattox, J. Adams, A.S. Rosenthal, R. Faanes, R. J. Eckner, R. A. Koup, J.L. Sullivan, Science 1990, 250, 1411.

[2] K. D. Hargave, J.R. Proudfood, K. G. Grozinger, E. Cullen, S. R. Kapadia, U. R. Patel, V. U. Fuchs, S. C. Mauldin, J. W. Vitous, D. W. Neil, J.M. Rose, G. C. Chow, M. T. Skoog, J. C. Wu, G. Schmidt, W. W. Engel, W. G. Eberlein, T. D. Saboe, S. J. Campell, A. S. Rosenthal, J. Adam, J. Med. Chem. 1991, 34, 2231. $3.51-3.60,3.90-3.99\left(2 \mathrm{~m}, 2 \mathrm{H}, \mathrm{NCH}_{2}\right), 4.88-4.93(\mathrm{~m}, 2 \mathrm{H}$, $\left.3^{\prime}-\mathrm{H}_{\mathrm{a}}, 3^{\prime}-\mathrm{H}_{\mathrm{b}}\right), 5.53-5.69\left(\mathrm{~m}, 1 \mathrm{H}, 2^{\prime}-\mathrm{H}\right), 7.18-7.81(\mathrm{~m}, 9 \mathrm{H}$, Ar-H). $-{ }^{13} \mathrm{C}$ NMR: $\delta=23.8,23.9\left(2 \mathrm{CH}_{3}\right), 41.9\left(\mathrm{NCH}_{2}\right)$, $66.1(\mathrm{OCH}), 91.3(\mathrm{C}-3), 117.4,133.5\left(\mathrm{CH}_{2}=\mathrm{CH}\right), 122.8$, 123.2, 123.8, 126.7, 128.4, 129.4, 130.4, 132.2, 138.7, 146.4 (Ar-C), $168.1(\mathrm{C}=\mathrm{O})$ ppm. - $\mathrm{C}_{20} \mathrm{H}_{21} \mathrm{NO}_{2}$ (307.4): calcd. C 78.15, H 6.89, N 4.56; found C 77.9, H 6.6, N 4.3.

\section{2-Allyl-3-methoxy-3-phenyl-2,3-dihydro-isoindol-1-one (11e)}

From 1b and methyl alcohol, m.p. $114{ }^{\circ} \mathrm{C}$; yield: $1.30 \mathrm{~g}(93 \%)$. - IR (KBr): $v=1614(\mathrm{C}=\mathrm{C}), 1687 \mathrm{~cm}^{-1}$ $(\mathrm{C}=\mathrm{O}) .-{ }^{1} \mathrm{H}$ NMR $\left(\mathrm{CDCl}_{3}\right): \delta=2.98\left(\mathrm{~s}, 3 \mathrm{H}, \mathrm{OCH}_{3}\right)$, $3.61-3.69,3.96-4.06\left(2 \mathrm{~m}, 2 \mathrm{H}, \mathrm{NCH}_{2}\right), 4.97-5.12(\mathrm{~m}, 2 \mathrm{H}$, $\left.3^{\prime}-\mathrm{H}_{\mathrm{a}}, 3^{\prime}-\mathrm{H}_{\mathrm{b}}\right), 5.67-5.80\left(\mathrm{~m}, 1 \mathrm{H}, 2^{\prime}-\mathrm{H}\right), 7.13-7.89(\mathrm{~m}$, 9H, Ar-H). $-{ }^{13} \mathrm{C}$ NMR: $\delta=41.0\left(\mathrm{NCH}_{2}\right), 49.4\left(\mathrm{OCH}_{3}\right)$, $94.4(\mathrm{C}-3), 116.8,131.6\left(\mathrm{CH}_{2}=\mathrm{CH}\right), 121.9,122.3,125.3$, 127.3, 127.4, 128.6, 131.4, 131.6, 137.6, 144.2 (Ar-C), 166.9 $(\mathrm{C}=\mathrm{O})$ ppm. $-\mathrm{C}_{18} \mathrm{H}_{17} \mathrm{NO}_{2}$ (279.4): calcd. C 77.37, H 6.09, N 5.00; found C 77.5, H 5.7, N 4.6.

\section{2-Allyl-3-ethoxy-3-phenyl-2,3-dihydro-isoindol-1-one (11f)}

From $\mathbf{1 b}$ and ethyl alcohol, m.p. $134{ }^{\circ} \mathrm{C}$; yield: $1.33 \mathrm{~g}$ (91\%). - IR (KBr): $v=1611(\mathrm{C}=\mathrm{C}), 1701 \mathrm{~cm}^{-1}(\mathrm{C}=\mathrm{O})$. ${ }^{1} \mathrm{H} \mathrm{NMR}\left(\mathrm{CDCl}_{3}\right): \delta=1.18\left(\mathrm{t}, 3 \mathrm{H}, J=7.1 \mathrm{~Hz}, \mathrm{CH}_{3}\right)$, $2.93-2.99\left(\mathrm{~m}, 2 \mathrm{H}, \mathrm{OCH}_{2}\right), 3.58-3.69,3.99-4.09(2 \mathrm{~m}$, $\left.2 \mathrm{H}, \mathrm{NCH}_{2}\right), 5.00-5.13\left(\mathrm{~m}, 2 \mathrm{H}, 3^{\prime}-\mathrm{H}_{\mathrm{a}}, 3^{\prime}-\mathrm{H}_{\mathrm{b}}\right), 5.69-5.85$ $\left(\mathrm{m}, 1 \mathrm{H}, 2^{\prime}-\mathrm{H}\right), 7.14-7.90(\mathrm{~m}, 9 \mathrm{H}, \mathrm{Ar}-\mathrm{H}) .-{ }^{13} \mathrm{C}$ NMR: $\delta=14.5\left(\mathrm{CH}_{3}\right), 41.9\left(\mathrm{NCH}_{2}\right), 58.0\left(\mathrm{OCH}_{2}\right), 94.4(\mathrm{C}-3)$, 117.4, $132.8\left(\mathrm{CH}_{2}=\mathrm{CH}\right), 122.8,123.1,126.1,128.1,128.2$, 129.3, 132.1, 138.7, $145.6($ Ar-C), $167.7(\mathrm{C}=\mathrm{O})$ ppm. $\mathrm{C}_{19} \mathrm{H}_{19} \mathrm{NO}_{2}$ (293.4): calcd. C 77.79, H 6.33, N 4.77; found C 77.4, H 6.5, N 4.3 .

\section{Acknowledgements}

The Deutsche Forschungsgemeinschaft (DFG) is gratefully acknowledged for partial support and Prof. Dr. Claus Nielsen for the determination of the biological activity against HIV.

[3] D. L. Romero, R. A. Morge, M. J. Genin, C. Biles, M. Busso, L. Resnick, I. W. Althaus, F. Reusser, R. C. Thomas, W. G. Tarpley, J. Med. Chem. 1993, 36, 1505.

[4] D. L. Romero, R. A. Morge, C. Biles, N. Berrios-Pena, P. D. May, J. R. Palmer, P.D. Jonson, H.W. Smith, M. Busso, C. K. Tan, R. L. Voorman, F. Reusser, I. W. Althaus, K. M. Downey, A. G. So, L. Resnick, W. G. Tarpley, P. A. Aristoff, J. Med. Chem. 1994, 37, 999.

[5] S. D. Young, S. F. Britcher, L. O. Tran, L. S. Payne, W. C. Lumma, T. A. Lyle, J. R. Huff, P. S. Anderson, D. B. Olsen, S. S. Caroll, D. J. Pettibone, J. A. O'Brien, 
R. G. Ball, S. K. Balani, J. H. Lin, I.-W. Chen, W. A. Scheif, V. V. Sardana, W. J. Long, V. W. Byrens, E. A. Emini, Antimicrob. Agents Chemother. 1995, 39, 2602.

[6] H. J. Stellbrink, Eur. J. Med. Res. 2007, 12, 483.

[7] K. Das, A. D. Clark, P. J. Lewi, J. Heeres, M. R. De Jonge, L. M. Koymans, H. M. Vinkers, F. Daeyaert, D. W. Ludovici, M. J. Kukla, B. De Corte, R. W. Kavash, C. Y. Ho, H. Ye, M. A. Lichtenstein, K. Andries, R. Pauwels, M.P. De Be'thune, P.L. Boyer, P. Clark, S.H. Hughes, P. A. Janssen, E. Arnold, J. Med. Chem. 2004, 47, 2550.

[8] M. M. Baba, S. Shigeta, S. Yuasa, H. Takashima, K. Sekiya, M. Ubasawa, H. Tanaka, T. Miyasaka, R. T. Walker, E. De Clercq, Antimicrob. Agents Chemother. 1994, 38, 688.

[9] R. Pauwels, K. Andries, Z. Debyser, M. J. Kukla, D. Schols, H.J. Breslin, R. Woestenborghs, J. Desmyter, M. A.C. Janssen, E. De Clercq, P. A. J. Janssen, Antimicrob. Agents Chemother. 1994, 38, 2863.

[10] A. A. Hamed, Z. Naturforsch. 2004, 56b, 589.

[11] A. A. Hamed, J. Chem. Res. (S) 2005, 54.

[12] C. Ahgren, K. Backro, F.W. Bell, A.S. Cantrell, M. Clemens, J. M. Colacino, J. B. Deeter, J. A. Engelhardt, M. Högberg, S. R. Jaskunas, N. G. Johansson, C. L. Jordan, J. S. Kasher, M. D. Kinnick, P. Lind, C. Lopez, J. M. Morin, M. A. Muesing, R. Noreen, B. Öberg, C. J. Paget, J. A. Palkowitz, C. A. Parrish, P. Pranc, M. K. Rippy, C. Rydergard, C. Sahlberg, S. Swanson, R. J. Ternansky, T. Unge, R. T. Vasileff, L. Vrang, S. J. West, H. Zhang, X.X. Zhou, Antimicrob. Agents Chemother. 1995, 39, 1329.
[13] G. Maass, U. Immendoerfer, B. Koenig, U. Leser, B. Mueller, R. Goody, E. Pfaff, Antimicrob. Agents Chemother. 1993, 37, 2612.

[14] A. E. Ismail, A. A. Hamed, M. T. Abdel-Aal, I. Zeid, M. Al-Talib, Q. Wang, J. C. Jochims, J. Prakt. Chem. 1992, 334, 661.

[15] C. Sahlberg, R. Noreen, P. Engelhardt, M. Högberg, J. Kangasmetsa, L. Vrang, H. Zhang, Bioorg. Med. Chem. Lett. 1998, 8, 1511.

[16] M. Högberg, C. Sahlberg, P. Engelhardt, R. Noreen, J. Kangasmetsa, N. G. Johansson, B. Öberg, L. Vrang, H. Zhang, B. L. Sahlberg, T. Unge, S. Lövgren, K. Fridborg, K. Backbro, J. Med. Chem. 1999, 42, 4150.

[17] M. Popovic, M. G. Sarngadharan, E. Read, R. C. Gallo, Science 1984, 224, 497.

[18] S. Harada, Y. Koyanagi, N. Yamamoto, Science 1985, $229,563$.

[19] C. M. Nielsen, I. C. Bygbjerg, B. F. Vestergaard, Lancet 1987, 1, 566.

[20] F. W. Bell, A. S. Cantrell, M. Högberg, S. R. Jaskunas, N. G. Johansson, C. L. Jordan, M. D. Kinnick, P. Lind, J. M. Morin, Jr., R. Noreen, B. Öberg, J. A. Palkowitz, C. A. Parrish, P. Pranc, C. Sahlberg, R. J. Ternansky, R. T. Vasileff, L. Vrang, S. J. West, H. Zhang, X.-X. Zhou, J. Med. Chem. 1995, 38, 4929.

[21] R. Vig, C. Mao, T. K. Venkatachalam, L. Tuel-Ahlgren, E. A. Sudbeck, F. M. Uckun, Bioorg. Med. Chem. 1998, 6, 1789.

[22] T. Price, L. R. Creagh, L. Truitt, J. Med. Chem. 1965, 8,731 .

[23] J. B. Campbell, R. F. Dedinas, S. A. Trumbowerwalsh, J. Org. Chem. 1996, 61, 620. 